\title{
铁载体分子偶联抗生素药物研究进展
}

\author{
刘君 侯劲松 孟影缪志颖 林 静陈卫民* \\ (暨南大学药学院 广州 510632)
}

\begin{abstract}
摘要 天然铁载体是细菌分泌的一种小分子铁离子螯合剂, 与铁离子螯合后可被特定的外膜受体识别并转运至胞浆内 为细菌提供必需的铁. 利用铁载体分子这种特性, 可将药物分子与其偶联, 通过细菌铁摄取系统使抗生素进入细菌从 而杀死细菌，这一策略被称为“特洛伊木马”策略. 2019 年，第一个铁载体-抗生素偶联药物 Cefiderocol 被批准上市，引 起许多专家和制药企业对本领域的研究兴趣. 从铁载体分子的类别、不同作用机制的抗生素以及连接体的选择三个方 面对铁载体分子偶联抗生素药物进行了较全面的综述, 总结了铁载体-抗生素偶联物其三个组成部分分别对于新抗生 素发挥抗菌作用的规律, 为新型铁载体偶联抗生素药物的研发提供参考.
\end{abstract}

关键词 抗生素; 铁载体分子; 偶联物; “特洛伊木马” 策略

\section{Research Progress of Antibiotics Conjugated with Siderophores}

\author{
Liu, Jun Hou, Jinsong Meng, Ying Miao, Zhiying Lin, Jing Chen, Weimin* \\ (College of Pharmacy, Jinan University, Guangzhou 510632)
}

\begin{abstract}
The natural siderophore is a class of small-molecule iron ion chelating agents secreted by bacteria, which can be recognized by specific outer membrane receptors and transported into cytoplasm to provide iron for bacteria. Using this characteristic of siderophore, antibiotics can be coupled with them and enter into bacteria through the bacterial iron uptake system. This strategy is called "Trojan horse" strategy. Recently, cefiderocol, the first siderophore-antibiotic conjugate, was approved for marketing, which has aroused accumulated interest of scientists and pharmaceutical companies in this field. This paper provides a comprehensive review of the progress in antibiotics conjugated with siderophores from three aspects: the types of siderophore molecules, antibiotics with different action mechanisms, and the role of linkers. The basic relationship between anti-bacterial activity and three moieties of this novel type of anti-bacterial agents has been revealed. This review will provide a reference for the development of new antibiotics conjugated with siderophores.
\end{abstract}

Keywords antibiotic; siderophore; conjugate; "Trojan horse" strategy

20 世纪 40 年代，抗生素被视为 “神奇药物” [1-5], 它优异的杀菌功效和较强的选择性一度使人们认为感 染性疾病将成为过去. 但是, 随着抗生素的广泛使用, 由于细菌产生耐药性而导致的感染治疗失败, 对人类健 康和生存构成了重大威胁 ${ }^{[5]}$, 因此临床上迫切需要新型 抗生素和抗菌策略来解决这一问题.

20 世纪 80 年代, Miller 团队提出 “特洛伊木马” 分 子抗生素策略, 将抗生素与天然铁载体或合成铁载体分 子偶联, 利用细菌自身的铁载体转运系统靶向将抗生素 递送到抗菌作用靶点 ${ }^{[6]}$. 这种策略的目的是促进抗生素 进入细菌细胞, 从而增加其抗菌活性或扩大其抗菌谱.
铁是几乎所有生命形式的重要营养素, 在所有生物中都 起着重要作用. 大多数的生物都需要铁, 它不仅是氧化 还原酶等重要生物酶的辅助因子, 也是参与氧气运输、 电子传递、能量产生和其它重要代谢过程的生物必需元 素 ${ }^{[7]}$. 在生理 $\mathrm{pH}(7.35 \sim 7.40)$ 条件下，人血清中的铁以 $\mathrm{Fe}^{3+}$ 形式存在, 约为 $10^{-24} \mathrm{~mol} / \mathrm{L}$, 而每个细菌细胞分裂 需要约 $10^{-5} \sim 10^{-6} \mathrm{~mol} / \mathrm{L} \mathrm{Fe}^{3+}$ (细胞内至少维持 $10^{-6}$ $\mathrm{mol} / \mathrm{L}$ )浓度. 为了在感染期间获得足够的铁来维持自身 生长并完成各种生理活动, 细菌进化出了一系列能够摄 取铁的系统，通过合成一系列小的有机化合物铁载体 (分子量在 $200 \sim 2000$ 之间), 并将其分泌到胞外, 利用

\footnotetext{
* Corresponding author. E-mail: twmchen@jnu.edu.cn

Received June 21, 2020; revised August 20, 2020; published online August 26, 2020.

Dedicated to Professor Henry N. C. Wong on the occasion of his 70th birthday.

Project supported by the National Natural Science Foundation of China (No. 81872776).

国家自然科学基金(No. 81872776)资助项目.
} 
主动转运系统从胞外获取各种形式的铁来维持生命活 动 ${ }^{[8]}$ (图 1).

已经发现的天然铁载体-抗生素偶联物有链需菌和 放线菌产生的阿波霉素、铁霉素和沙霉素等 ${ }^{[9]}$. 阿波霉 素和沙霉素是研究较多的两类天然铁载体-抗生素偶联 物, 已经证明它们可使用特定的铁转运系统进入目标细

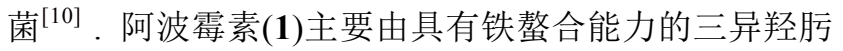
酸部分和抗菌的硫代核糖嘧啶部分(图 2)组成, 在阴性 菌大肠杆菌内, 阿波霉素通过 TonB 依赖性转运蛋白 $\mathrm{FhuA}^{[11]}$ (外膜识别)、FhuD ${ }^{[12]}$ (周质)、FhuB(细胞质膜)
和 $\mathrm{FhuC}$ (一种 ATPase, 为跨内膜转运提供能量)主动转 运至细胞质中。一旦阿波霉素进入细胞质，就会被一种 肽酶裂解，在细胞质中释放抗菌成分硫代核糖嘧啶，通 过抑制氨酰基-tRNA 合成酶以阻断蛋白质合成发挥抗 菌作用. 沙霉素(2)(图 2) 由三异羟肟酸酯和氨基糖苷两 个部分组成, 沙霉素可以通过铁还原来引发分子内环化 作用释放其氨基糖苷部分来抑制蛋白质合成发挥抗菌 作用 ${ }^{[13]}$. 这些天然铁载体-抗生素偶联物, 通过借助铁 离子吸收系统进入到细菌体内，进而将药物分子传递到 胞内作用靶点来发挥抗菌作用。这些天然铁载体一

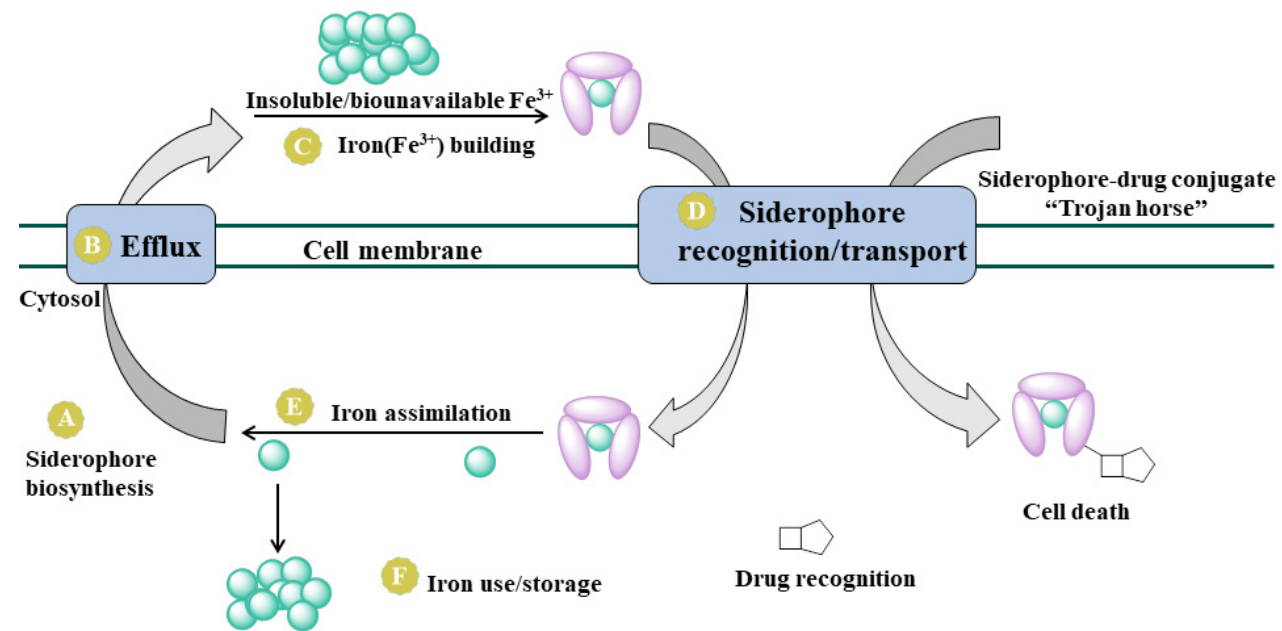

图 1 铁载体介导的铁转运系统及 “特洛伊木马” 策略 ${ }^{[8]}$

Figure 1 Siderophore-mediated iron acquisition and its exploitation by "Trojan horses"

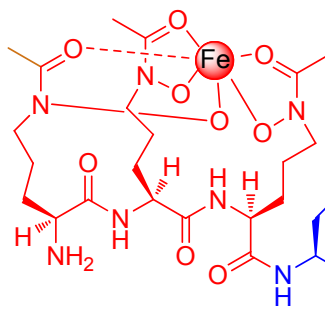

1 Albomycins

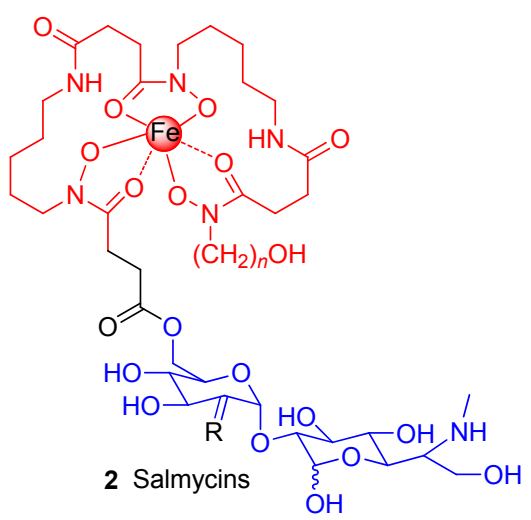

Peptidase in cytoplasm $\underset{\text { (ii) Cyclization }}{\stackrel{\text { (i) }}{\longrightarrow}}$

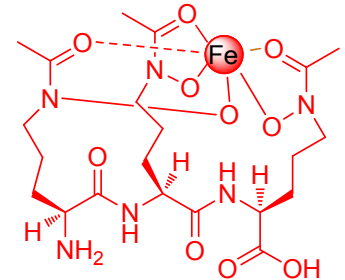

$\mathrm{R}=\mathrm{O}, \mathrm{NH}, \mathrm{NCONH}_{2}$<smiles></smiles><smiles>CN(O)C(=O)CCC(=O)NCCCCCN(O)C(=O)CCC(=O)NCCCCCN1OC(=O)CCC1=O</smiles>

图 2 天然铁霉素释放抗生素的过程 ${ }^{[15 ~ 17]}$

Figure 2 Antibiotics released from natural sideromycins 
抗生素偶联物的发现鼓舞科研人员利用 “特洛伊木 马” 分子抗生素策略来设计新型具有抗菌作用的化合 物. 2019 年 11 月 14 日, 美国食品药品监督管理局(FDA) 批准了一个铁载体分子偶联抗生素药物头孢地尔 (Cefiderocol)上市, 进一步验证了 “特洛伊木马” 分子 抗生素策略可以作为一种靶向传递抗生素分子来解决 细菌耐药问题的可行性 ${ }^{[14]}$. 但在已有的文献中, 偶联物 发挥抗菌作用有成功的例子也有失败的例子, 什么样的 抗生素与什么样的铁载体分子偶联可以达到 “特洛伊 木马” 策略的效果? 还没有此类药物分子设计的指引. 在本文中, 我们将详细总结分析已有文献中不同作用机 制抗生素与铁载体分子偶联对抗菌活性的影响, 试图找 出一些规律, 为新型铁载体分子偶联抗生素药物研发提 供参考.

\section{1 铁载体分子}

迄今为止，已经有超过 500 种铁载体在细菌、真菌、 植物中被发现 ${ }^{[18]}$. 铁载体通常被细菌合成并释放到环 境中, 可以极高的亲和力螯合 $\mathrm{Fe}^{3+}, \mathrm{Fe}^{3+}$-铁载体螯合物 可被细菌的特定膜蛋白受体识别, 从而将螯合物转运至 细菌内部. 一旦螯合物进入到细菌内的还原性细胞质 中, $\mathrm{Fe}^{3+}$ 就会被还原为 $\mathrm{Fe}^{2+}$, 导致铁载体失去亲和力, 随 后释放的 $\mathrm{Fe}^{2+}$ 被细菌利用 ${ }^{[19]}$. 铁载体根据来源主要分为 两大类, 天然铁载体和人工合成类铁载体. 天然铁载体 是由细菌、真菌或植物分泌出的铁载体 ${ }^{[9,20]}$, 例如革兰 氏阳性菌金黄色葡萄球菌(Staphylococcus aureus) 分泌的 葡萄铁蛋白 ${ }^{[21]}($ Staphyloferrin, 3)(图 3)、革兰氏阴性菌的 大肠杆菌(E. coli)分泌的肠杆菌素 ${ }^{[22]}$ (Enterobactin, 4)(图
3)、铜绿假单胞菌(Pseudomonas aeruginosa)分泌的苂光 嗜铁素(Pyoverdine, 5)和螯铁蛋白 ${ }^{[23]}$ (Pyochelin, 6)(图 3), 还有从果蝇红酵母菌 ${ }^{[24]}$ (Rhodotorula pilimanae)中提取 出来的红酵母酸(Rhodotorulic acid, 7)(图 3)和镰刀菌 (Fusarium roseum) 产生的一种线型 Fusarinine B (8) ${ }^{[25]}$ 铁 载体(图 3)等. 这些天然铁载体以极高的亲和力与三价 铁离子结合形成螯合物，被相应的受体识别并转运进入 胞内, 为生物提供铁离子, 从而提供竞争性的生长优势.

天然铁载体具有高度多样化的结构，根据铁螯合功 能基团的化学性质，天然铁载体可以分为三大类：异羟 肜酸型、儿茶酚型和 $\alpha$-差基羧酸型 ${ }^{[26-27]}$. 异差肟酸型 (9)(图 4)是自然界中最普遍存在的铁载体, 大部分由细 菌真菌等微生物产生. 其 $N$-羟基和羰基氧原子作为 $\mathrm{Fe}^{3}$ ${ }^{+}$的配体, 与 $\mathrm{Fe}^{3+}$ 的结合常数大约在 $10^{22} \sim 10^{32}$ 之间, 这 种高亲和力的结合可以保护螯合物免受水解和酶促的 降解. 儿茶酚型(10)(图 4)则一般由一些细菌产生, 因为 它们具有邻苯二酚结构, 所以苯环上相邻的两个羟基能 够作为 $\mathrm{Fe}^{3+}$ 的配体 ${ }^{[28]} ; \alpha$-差基羧酸型(11)铁载体(图 4)则 通常由细菌和真菌(毛霉菌)产生，通过羧基和羟基与 $\mathrm{Fe}^{3+}$ 结合. 合成类铁载体的最常见的结构主要也是以异 着肟酸、儿茶酚和 $\alpha$-羟基羧酸, 以及类儿茶酚结构的着弪 基吡啶酮(12)为主要骨架 ${ }^{[29-31]}$, 这些基团均可以与 $\mathrm{Fe}^{3+}$ 形成六齿配位的稳定结构。这些天然及合成类铁载体借 助铁特异性摄取系统, 可以促使与其连接的抗生素靶向 输送至病原体特定作用靶点, 有助于抗生素跨过细菌细 胞膜. 对于具有复杂结构细胞壁的革兰氏阴性病原体, 抗生素可以借助铁载体的转运，克服外膜通透性降低而 产生的细菌耐药性.<smiles>NC(CNC(=O)C[C@H](CC(=O)NCCNC(=O)CCC(=O)C(=O)O)C(=O)O)C(=O)O</smiles>

3 Staphyloferrin<smiles>O=C(N[C@@H](COC(=O)[C@H](COC(=O)[C@H](COC(=O)c1cccc(O)c1O)NC(=O)c1cccc(O)c1O)NC(=O)c1cccc(O)c1O)C(=O)O)c1cccc(O)c1O</smiles>

4 Enterobactin

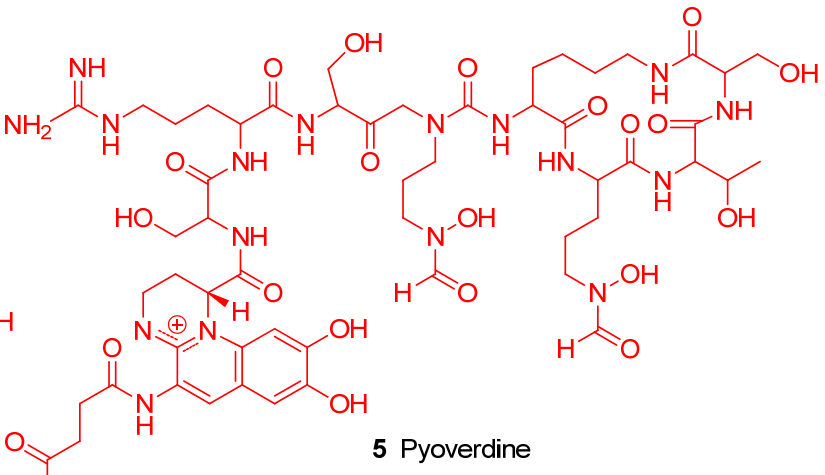

$\mathrm{OH}$

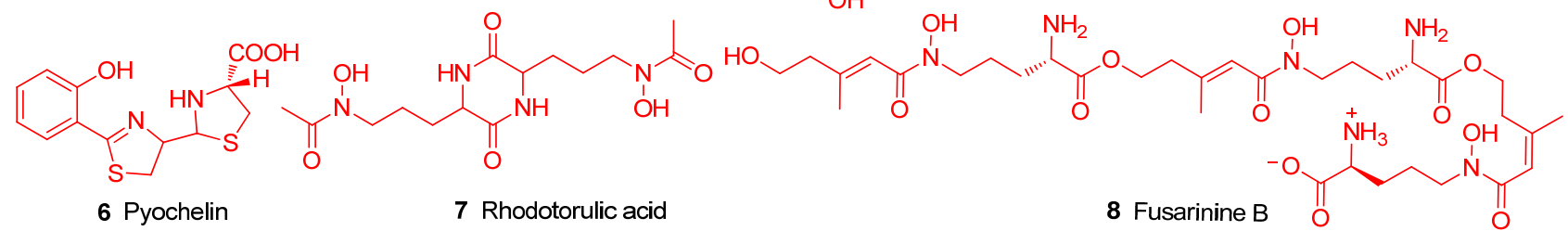

图 3 天然铁载体的结构

Figure 3 Structures of natural siderophores 
<smiles>[R]C(=O)N([R])O</smiles>

9 Hydroxamate<smiles>[R]C([R])(O)C(=O)O</smiles>

11 Hydroxycarboxylate<smiles>[R]c1cccc(O)c1O</smiles>

10 Catecholate<smiles>[R]c1cc(=O)c(O)cn1CC</smiles>

12 3-Hydroxypyridone

图 4 载铁体络合铁官能团结构单元

Figure 4 Structural units of iron complexes and functional groups

\section{2 以细菌细胞壁为靶标的抗生素与铁载体偶联 物的研究}

作用于细菌细胞壁的抗生素-铁载体类偶联物的药 物研发可追溯到 20 世纪 80 年代, Benz 和同事们 ${ }^{[32]}$ 发现 $^{\circ}$ 阿波霉素中的 $N^{5}$-乙酰基- $N^{5}$-差基- $L$-鸟氨酸基团是细菌 参与铁转运系统铁载体的必要基团. 1990 年, Miller 课题 组 ${ }^{[32]}$ 以 $N^{5}$-乙酰基- $N^{5}$-羟基- $L$-鸟氨酸作为铁载体与第一 代 $\beta$-内酰胺类头孢菌素氯碳头孢(loracarbef)偶联得到偶 联物 13 和 14(图 5), 来探究 $\beta$-内酰胺类抗生素偶联物的 抗菌活性. 初步活性结果表明, 偶联物 13 和 14 在 1 和 $10 \mu \mathrm{mol} / \mathrm{L}$ 时均有效地抑制了大肠杆菌的生长, 进一步 证实 $\beta$-内酰胺类抗生素有望作为 “特洛伊木马” 分子 策略中的抗生素进行深入研究. 遗憾的是, 偶联物 13 、

14 相比母体抗生素氯碳头孢的抗菌活性没有显著提升. 随后, Miller 课题组 ${ }^{[33-34]}$ 进一步探究了不同类型铁载体, 异羟肟酸和儿茶酚铁载体与氯碳头孢偶联物 $(15,16)$ (图 5)的抗菌活性. 初步活性结果表明, 异差肟酸酯铁载体 偶联物 15 和儿茶酚铁载体偶联物 16 可以分别利用不同 的外膜受体蛋白 $\mathrm{Fhu}$ 和 $\mathrm{Cir}$ 进入周质释放氯碳头孢抗生 素, 并与细菌内青霉素结合蛋白 (PBPs) 直接相互作 用 ${ }^{[35]}$, 发挥优秀的大肠杆菌抗菌活性. 但遗憾的是, 这 些偶联物分别只能作用于具有 Fhu 和 Cir 膜蛋白受体的 大肠杆菌来发挥抗菌活性, 对于 Fhu 和 Cir 膜蛋白缺陷 型受体的细菌菌株无效, 其无法借助铁载体铁转运系统 进入到细菌细胞提高杀菌活性. 所以, Miller 课题组 ${ }^{[36]}$ 进一步设计并合成一种以氯碳头孢作为抗菌成分, 结构 中具有儿茶酚和单异羟肟酸酯的混合型铁载体偶联物 17(图 5)来提升偶联物的抗菌活性. 初步活性探究发现, 17 可以利用大肠杆菌中多种铁载体的运输途径来发挥 抗菌活性, 不仅对大肠杆菌株有效, 对几种膜受体突变 类型的菌株也均有显著的抗菌活性. 此外, 偶联物 17 对 耐甲氧西林金黄色葡萄球菌(MRSA $)^{[37]}$ 的最小抑菌浓度

(Minimum Inhibitory Concentration, MIC) 值仅为 $2 \sim 8$ $\mu \mathrm{g} / \mathrm{mL}$, 其抗菌活性更优于母体抗生素氯碳头孢(MIC 值 $>128 \mu \mathrm{g} / \mathrm{mL})$. 这些研究表明相较于天然铁载体中的 $N^{5}$-乙酰基- $N^{5}$-着基- $L$-鸟氨酸基团和单一合成类铁载体, 混合类铁载体儿茶酚和异羟肟酸酯, 在规避常见的抗生 素耐药机制, 如外膜通透性障碍和外排机制等方面更为 有效，具有更优秀的抗菌活性. 所以，合成类混合型铁 载体与抗生素偶联物展现的优秀抗菌活性为未来 “特 洛伊木马” 分子抗生素策略的铁载体选择提供了新发 展方向.

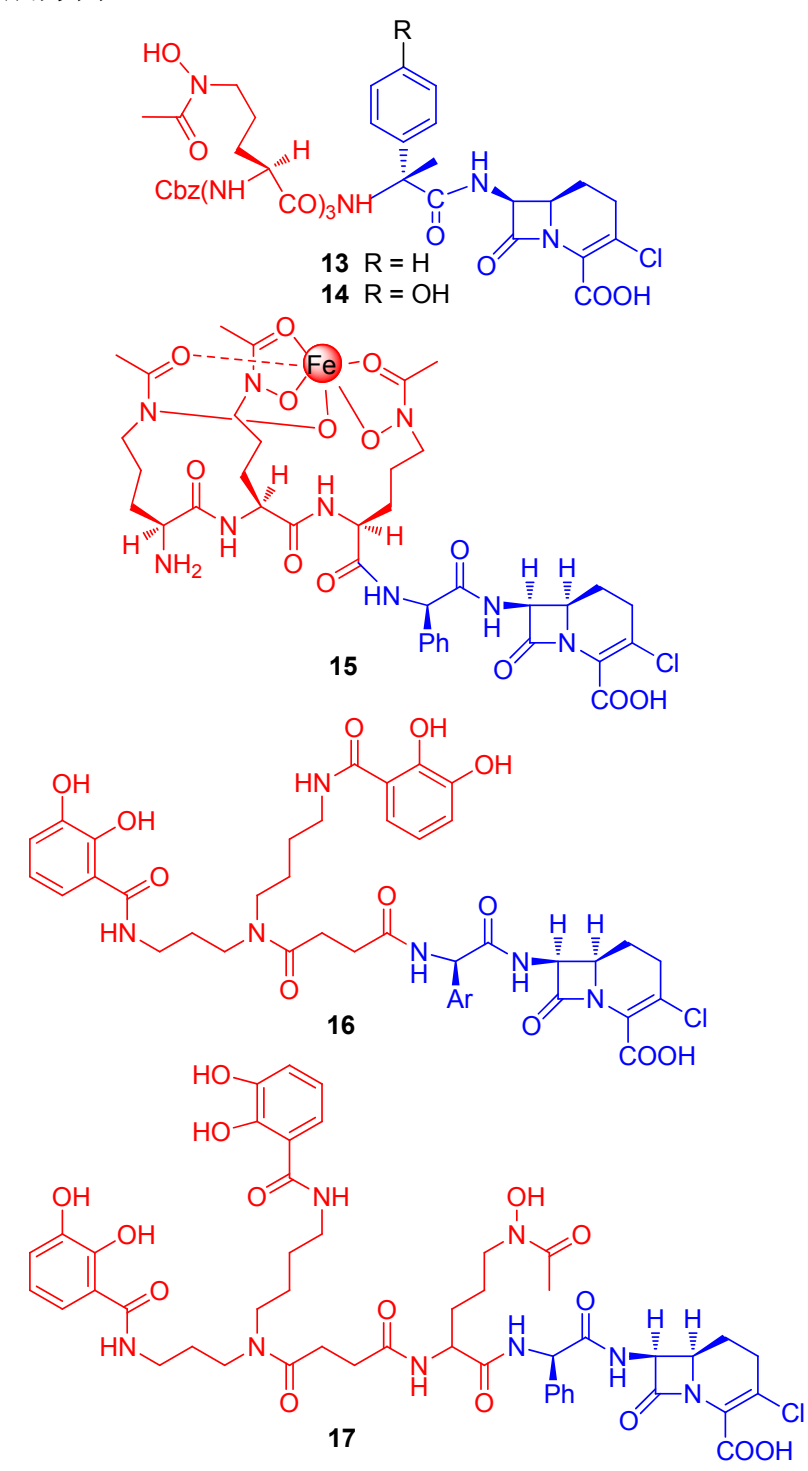

图 5 氯碳头孢一铁载体类偶联物

Figure 5 Loracarbef-siderophore conjugates

为了进一步探究作用于细菌细胞壁的其它类抗生 素是否也可以作为 “特洛伊木马” 分子的抗生素, 1996 年 Miller 团队 ${ }^{[37]}$ 分别将万古霉素(Vancomycin)与亚精胺 基邻茶二酚、儿茶酚和异羟肟酸酯的混合型铁载体偶联, 得到偶联物 18 和 19(图 6). 初步活性结果表明, 亚精胺 
基邻苯二酚 18 和混合型铁载体偶联物 19 相比于母体抗 生素万古霉素, 丧失了对革兰氏阳性菌的抗菌活性. 所 以，后续的 “特洛伊木马” 分子抗生素策略研究没有再 使用万古霉素作为抗生素. 1998 年, Budzikiewicz 等 ${ }^{[38]}$ 合成了两种天然铁载体 I 型苂光嗜铁素与氨苄青霉素的 偶联物 20 和 II 型苂光嗜铁素与氨苄青霉素的偶联物 21(图 6). 该系列偶联物选择二羧酸作为 I 型苂光嗜铁素 和 II 型苂光嗜铁素中的 Lys 残基与抗生素之间的连接基 才, 这种长而灵活的连接基团可以避免氨茮青霉素与其 靶向的细菌转肽酶之间产生空间位阻, 使药物与铁载体 紧密结合而不影响外膜识别. 初步活性数据显示, 偶联 物 20 和 21 具有显著的铜绿假单胞菌抗菌活性, 偶联物
20 和 21 的 MIC 值分别为 0.39 和 $0.24 \mu \mathrm{mol} / \mathrm{L}$. 这表明 偶联物可以通过铁摄取途径进入细菌细胞, 在细菌内释 放氨芳青霉素，从而发挥抗菌活性. 遗憾的是该类型天 然铁载体仅对利用苂光嗜铁素类菌株有效，具有很大的 局限性.

2002 年, 为了启动基于铁载体的 “特洛伊木马” 策略抗生素最全面的研究, 德国汉诺尔研究所(HKI)的 Möllmann 研究小组 ${ }^{[39]}$ 合成了大量的青霉素与铁载体的 偶联物，主要是利用完全合成的仿生铁载体类似物与抗 生素偶联来探究偶联物的抗菌活性. 他们将人工合成双 儿茶酚类铁载体与氨苄青霉素偶联得到偶联物 22(图 7), 初步抗菌活性测试表明，与母体抗生素氨苄青霉素相

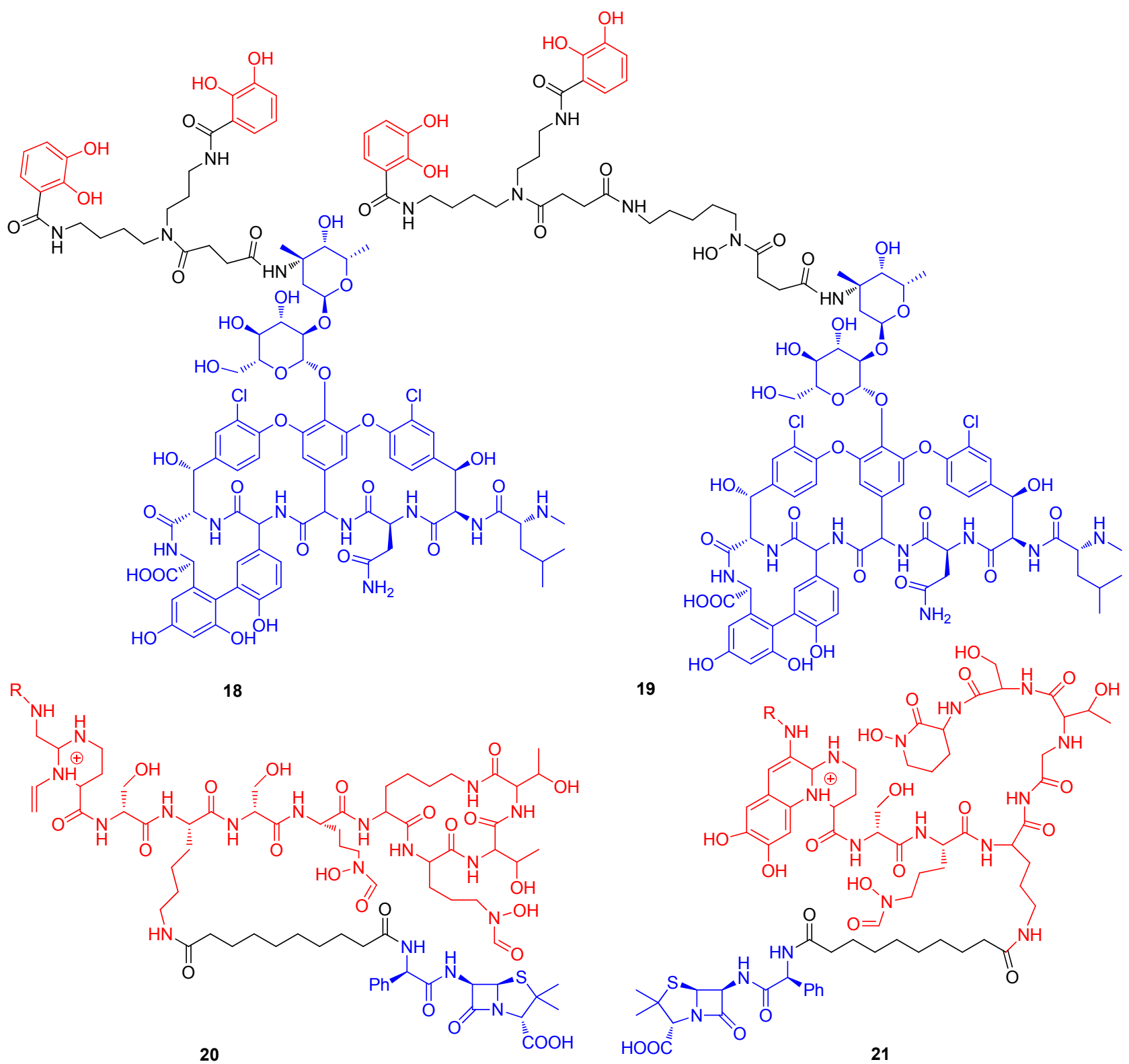

图 6 万古霉素和氨苄青霉素类-铁载体偶联物

Figure 6 Vancomycin and ampicillin-siderophore conjugates 
比, 偶联物 22 的铜绿假单胞菌菌株的抗菌能力提升 1000 倍以上 $(22$ 的 $\mathrm{MIC}<0.05 \mu \mathrm{g} / \mathrm{mL}$; 氨芐青霉素 $>100$ $\mu \mathrm{g} / \mathrm{mL}) .2003$ 年, Möllmann 研究小组 ${ }^{[40]}$ 进一步探究双儿 茶酚和异羟肜酸酯混合的铁载体与氨苄青霉素偶联后 偶联物 23(图 7) 的抗菌活性, 发现 23 不仅对革兰氏阴性 菌铜绿假单胞菌的抗菌活性比母体氨芳青霉素提升了 2000 倍, 对革兰氏阳性菌的抗菌活性也有所提升 $(23$ 对 金黄色葡萄球菌 MIC 值为 $0.4 \mu \mathrm{g} / \mathrm{mL}$; 氨苄青霉素 MIC 值为 $25 \mu \mathrm{g} / \mathrm{mL}$ ). 综上, 双儿茶酚和异羟肜酸酯类混合 型结构对于识别细菌铁载体至关重要. 由于混合型铁载 体具有可被细菌膜受体识别的优势, 2012 年, Miller 团 队 ${ }^{[41-42]}$ 设计并合成具有简化型的三脚骨架连接基的三 儿茶酚类铁载体与抗生素氨芐青霉素和阿莫西林偶联, 得到相应的偶联物 24 和 25 (图 7), 进一步探究三脚骨架 连接基的混合型铁载体对偶联物抗菌活性影响. 初步抗 菌活性发现, 24 和 25 对革兰氏阴性菌铜绿假单胞菌呈 现抗菌活性, 已有研究表明氨苄青霉素与阿莫西林本身 对野生型铜绿假单胞菌不具备抗菌活性( MIC $>200$ $\mu \mathrm{mol} / \mathrm{L}$ ), 但氨苄青霉素与阿莫西林与铁载体偶联后却 显现出优秀的抗菌活性, 尤其在缺铁培养基中, 其 MIC 值为 $0.05 \sim 0.39 \mu \mathrm{mol} / \mathrm{L}$. 早在 2009 年, Möllmann 研究小 组 ${ }^{[43]}$ 就阐述了合成类铁载体双儿茶酚的氨苄青霉素偶 联物的作用机制(22), 利用细菌铁转运系统, 绕过外排 洜进入到革兰氏阴性菌体内发挥抗菌活性. 更令人兴奋 的是, 该偶联物对小鼠具有良好的耐受性, 且没有诱变 作用. 这些结果表明, 与天然铁载体相比, 人工合成类 铁载体提高了以氨芳青霉素或阿莫西林作为抗生素的 偶联物抗菌活性, 尤其是混合型人工合成类铁载体的偶 联物具有更优的抗菌活性, 混合类合成铁载体可能是未 来的 “特洛伊木马” 分子抗生素策略中铁载体发展的 主要方向.

通过铁载体偶联物来增强 $\beta$-内酰胺类抗生素的尝 试, 不仅仅停留在青霉素和头孢菌素类抗生素. 氨曲南 26(图 8) 也可以作为 “特洛伊木马” 分子策略中的抗生 素, 且是临床上唯一使用的 ${ }^{[44]}$, 对 $\beta$-内酰胺酶更具抗性 的单环 $\beta$-内酰胺类抗生素 ${ }^{[45]} .1985$ 年, Squibb 医学研究 所 ${ }^{[46-47]}$ 报道了以氨曲南(26)作为抗菌成分, 咪唑啉酮为 连接部分, 美基吡啶酮基团作为铁载体的偶联物 27(图 $8)$ 的合成与研究. 虽然羟基吡啶酮不是天然的铁载体的 组成部分, 但它们通常被认为是儿茶酚类铁载体的模拟 结构, 并且具有高亲和性的铁螯合能力. 初步抗菌活性 显示, 与母体抗生素氨曲南的抗菌活性一样, 偶联物 27 对革兰氏阴性菌也具有显著的抗菌活性, 尤其对铜绿假 单胞菌菌株的抗菌活性有所提高 $(27$ 的 MIC 为 $0.5 \mu \mathrm{g} /$ $\mathrm{mL}$, 氨曲南 $\mathrm{MIC}$ 值为 $4 \mu \mathrm{g} / \mathrm{mL}$ ). 随后, Tuominen 小组 ${ }^{[47]}$

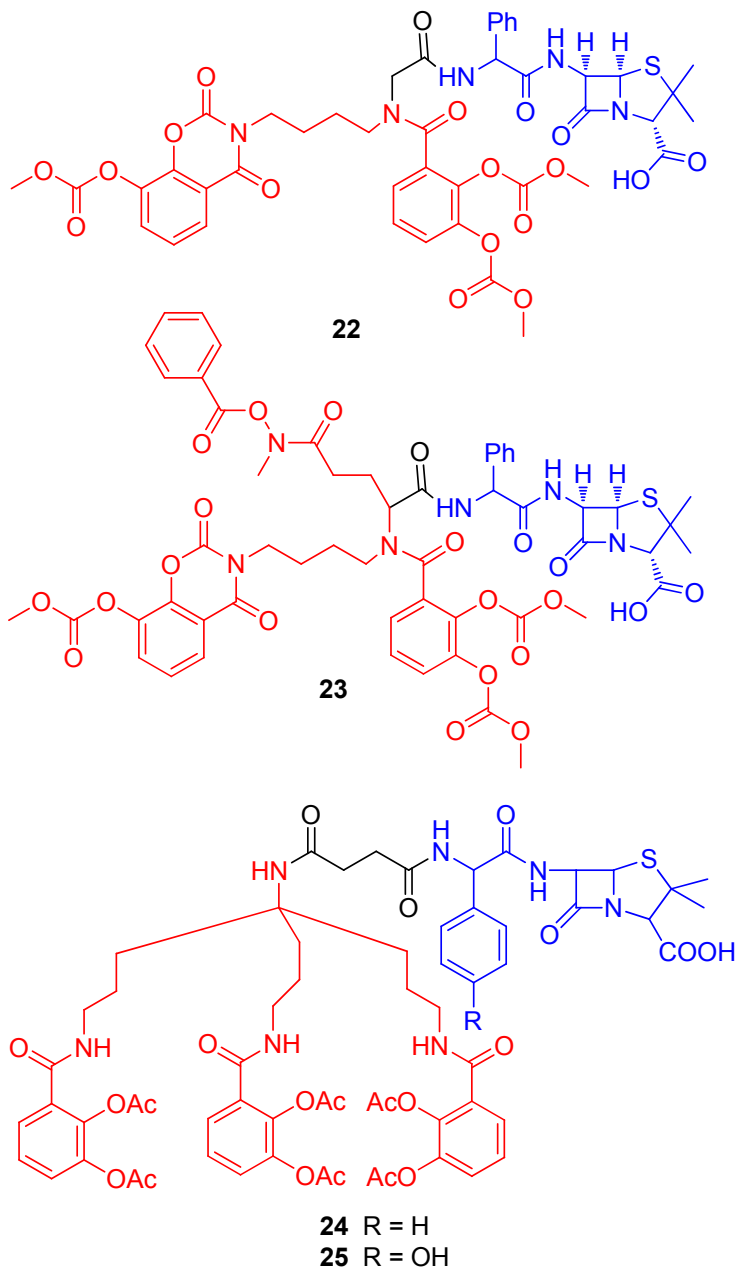

图 7 氨芳青霉素和阿莫西林-铁载体偶联物

Figure 7 Ampicillin and amoxicillin-siderophore conjugates

扩大了对该系列化合物连接部分的结构探究, 确定了结 构中以三唑酮作为连接部分, 具有强大的抗革兰氏阴性 菌的 U-78608 (28)先导化合物. 随着大型制药公司合并, 有关抗生素的研究工作也被削减. 直到 2011 年 ${ }^{[48-49]}$, 随 着抗生素的耐药性越来越普遍，一些研究人员或制药公 司(辉瑞公司)重新考虑了三唑酮作为连接部分的羟基吡 啶酮衍生物类 “特洛伊木马” 分子抗生素的研究, 设计 并合成偶联物 MC-1(图 8, 29) ${ }^{[50]}$, 主要是在 U-78608 (28) 结构基础上将三唑酮甲基替换为丙二醇. 初步体外活性 表明, MC-1 (29)对革兰氏阴性菌具有显著的抗菌活性, 与临床使用的氨曲南相比其抗菌活性显著提升, 对铜绿 假单胞菌的 MIC 值为 $0.5 \mu \mathrm{g} / \mathrm{mL}$. 进一步的机制研究表 明, 在铜绿假单胞菌的菌株中, MC-1 (29)利用了 PiuA 膜蛋白, 一种依赖 TonB 的铁载体外膜蛋白, 进入细菌 内发挥抗菌活性. 该小组还证明, MC-1 (29)对传统的低 通透性突变体也具有活性，可绕过细菌外排洜也不会被 大量 $\beta$-内酰胺酶水解进入细菌发挥抗菌活性. 作者得出 的结论是: “MC-1 可能是该系列中最具代表性的分子, 


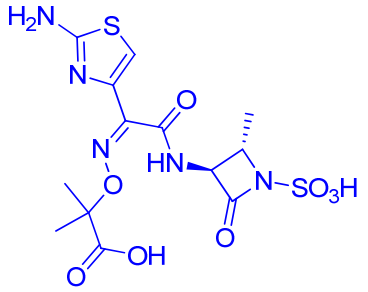

26 Aztreonam<smiles>C[C@H]1C(NC(=O)/C(=N\OC(C)(C)C(=O)O)c2csc(N)n2)C(=O)N1S(=O)(=O)N1CC(c2cc(=O)c(O)c[nH]2)N(C)C1=O</smiles>

27<smiles>C[C@H]1C(NC(=O)/C(=N\OC(C)(C)C(=O)O)c2csc(N)n2)C(=O)N1S(=O)(=O)n1nc(-c2cc(=O)c(O)c[nH]2)n(C)c1=O</smiles>

28 U-78608<smiles>C[C@H]1[C@H](NC(=O)/C(=N\OC(C)(C)C(=O)O)c2csc(N)n2)C(=O)N1C(=O)NS(=O)(=O)n1nc(-c2cc(=O)c(O)c[nH]2)n(C[C@H](O)CO)c1=O</smiles>

29 MC-1

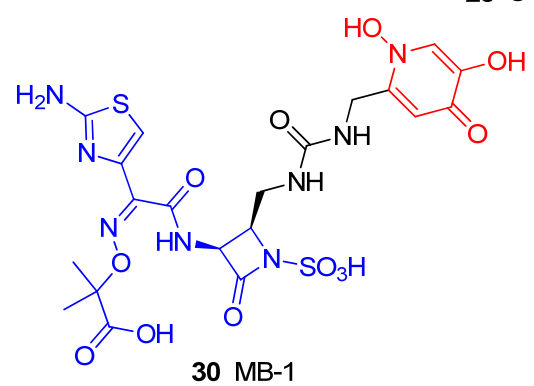

图 8 单环 $\beta$-内酰胺类抗生素-铁载体偶联物

Figure 8 Monocyclic $\beta$-lactam antibiotic-siderophore conjugates

它可以承受多种抗生素耐药机制的作用, 为临床医生与 铜绿假单胞菌等革兰氏阴性病原体的持续斗争提供了 武器” . 在此基础上, 辉瑞药业也报导了单酰胺羟基吡 啶酮 MB-1 (30) $)^{[51]}$ (图 8), 为了模拟 MC-1 中铁螯合剂基 团的位置, MB-1 结构中羟基吡啶酮连接在 C-4 官能化的 单环 $\beta$ 内酰胺上. 初步活性数据显示, 与 $\mathrm{MC}-1$ 一样, 新 的单环 $\beta$ 内酰胺 MB-1 偶联物也表现出优秀的革兰氏阴 性抗菌活性, 且不易产生耐药性. 综上, 单环 $\beta$ 内酰胺 类 “特洛伊木马” 分子抗生素也呈现优秀的抗菌活性, 为未来铁载体类抗生素的研发提供了另一个全新的方 向.

20 世纪 80 年代至今, 已有 cefetecol (32)、BAL30072 (31)、GSK3342830 和头孢地尔(33)(图 9)等 4 种 “特洛 伊木马” 分子抗生素进入临床试验 ${ }^{[2]}$. BAL30072 是一 种新型羟基吡啶酮与单环 $\beta$-内酰胺抗生素的偶联物. BAL30072 对铜绿假单胞菌和鲍曼不动杆菌均具有优秀 的抗菌活性 ( $\mathrm{MIC}_{90}$ 分别为 4 和 $8 \mu \mathrm{g} / \mathrm{mL}$ ), 但由于 BAL30072 严重肝毒性仅到 I 期临床. 头孢菌素 cefetecol ${ }^{[53]}$ 主要由于哺乳动物体内的儿茶酚 $O$-甲基转 移酶可使儿茶酚类铁载体的邻苯二酚上的 1 个酚基甲基 化，从而导致其体内活性下降也停止于 I 期临床. 2019 年 11 月 14 日 $^{[54]}$, 美国食品药品监督管理局(FDA)批准 日本盐野义公司研发的新型铁载体头孢菌素类抗生素 头狍地尔(Cefiderocol, Fetroja)上市. 该药物主要用于治 疗易感革兰氏阴性微生物引起的复杂性尿路感染的 18 岁及以上成人患者. 首个新型铁载体头孢菌素类抗生素 的成功, 为复杂性尿路感染患者带来了新的希望.

历时 30 多年, 日本盐野义制药有限公司完成了从<smiles>CC(C)(C)[14CH3]</smiles>

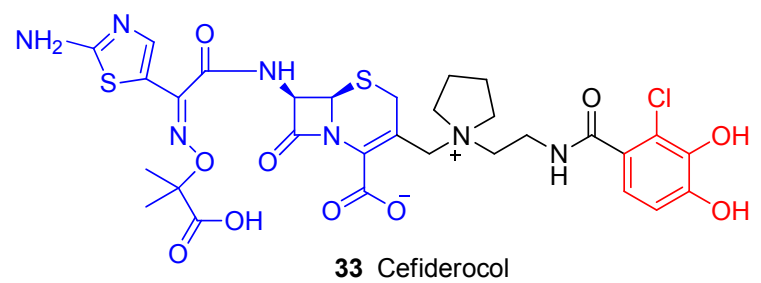<smiles>CC(C)(C(=O)O)C(=O)N=C(C(=O)NC1C(=O)N2C(C[N+]3(C)CCN(C(=O)c4ccc(O)c(O)c4)CC3)CSC12)c1csc(N)n1</smiles>

图 9 合成类铁载体结合 $\beta$-内酰胺和头孢菌素的结构 Figure 9 Structures of synthetic $\beta$-lactams and cephalosporins antibiotic-siderophore conjugates

铁载体类抗生素研发到头孢地尔铁载体类抗生素进入 市场的历程. 在 20 世界 90 年代初期，盐野义制药有限 公司发现铁载体头孢菌素 S-9096 (34) ${ }^{[55]}$ (图 9), 其对革 兰阴性菌(绿脓杆菌)具有强效的抗菌活性。该化合物 C-3 侧链上具有儿茶酚铁载体的结构, 可利用三价铁转 运系统对革兰氏阴性细菌的外膜具有良好的渗透性，从 而发挥优秀的抗菌活性. 但是，由于心血管毒性和化合 
物的稳定性差, S-9096 尚未到临床开发. 之后的 15 年里, 盐野义制药有限公司终止了对 “特洛伊木马” 策略抗 生素的研发. 随着抗生素的耐药性越来越普遍, 多家制 药公司和课题组对 $\beta$-内酰胺类 “特洛伊木马” 抗生素 展开深入的研究. 1992 年, 法国 ICI 制药公司通过对 BAL30072 引入氯原子得到 37(图 10)偶联物来降低邻苯 二酚部分的 $\mathrm{p} K_{\mathrm{a}}$ 值, 防止哺乳动物体内的儿茶酚 $O$-甲基 转移酶使其甲基化, 保留了铁载体的螯合特性, 除了具 有出色的抗菌活性, 37 更具有较长的药代动力学半衰 期. 遗憾的是, 由于缺乏针对 $\beta$-内酰胺酶的水解的稳定 性, 37 化合物未进入市场 ${ }^{[57]}$. 2008 年, 盐野义制药有限 公司重启探寻 “特洛伊木马” 分子抗生素之路并聚焦 于 $\beta$-内酰胺类抗生素与铁载体偶联物的研究. 十年之 后, 盐野义制药有限公司的 Aoki 等 ${ }^{[56]}$ 对头孢地尔抗生 素药物的设计与合成进行了报道, 详细描述了头孢地尔 的构效关系. 新型铁载体类头孢菌素头孢地尔是由氯儿 茶酚铁载体的部分、C-7 侧链引入了头孢他啶(35)(图 10) 酰氨基的部分和 C-3 侧链结合了头孢吡肟(36)(图 10)季 铵盐的部分组成. 头孢地尔抗生素中氯儿茶酚基团的铁 载体部分以偶联物 37 为基础设计, 可以避免铁载体不 被甲基转移酶甲基化, 保留铁载体螯合活性. C-3 侧链 上引入与头孢吡肜相似的吡咯烷基团, 提高了头孢地尔 对 $\beta$-内酰胺酶的稳定性发挥抗菌活性. 头狍地尔在 C-7 侧链上引入头孢他啶中羧基丙氧基亚氨基基团部分, 可 改善偶联物的跨外膜运输, 将抗生素转运至细胞周质发 挥抗菌活性. 铁载体、抗生素、连接基团三个部分的绝
对优化，让他们发现了新型 “特洛伊木马” 分子抗生素 头孢地尔. 头孢地尔的作用机制是与三价铁离子结合, 并通过细菌铁转运蛋白, 从细胞膜外膜被主动运输至细 菌外周胞质内通过抑制细胞壁生物合成所必需的青霉 素结合蛋白(PBPs), 导致细菌细胞死亡. 这种 “特洛伊 木马” 策略允许头孢地尔在细菌细胞外周胞质中达到 更高的浓度, 在外周胞质空间中与青霉素结合蛋白结合 并抑制细菌细胞壁的合成, 发挥抗菌活性.

综上, 可以发现天然铁载体与作用于细菌细胞壁类 抗生素偶联, 其抗菌活性大都很难优于母体抗生素, 而 且也容易出现其他挑战，如偶联物的溶解性低、穿过细 菌胞质膜的通道不足，以及活性抗生素的释放困难等问 题; 与天然铁载体相比, 合成类铁载体偶联作用于细菌 细胞壁类抗生素的抗菌活性显著增强, 其结构简单、靶 向特异性强和活性抗生素释放充足, 也更深受科学家和 制药公司的喜爱; 相较于其它作用机制类的抗生素，作 用靶标位于外周胞质空间内的细胞壁生物合成的青霉 素结合蛋白(PBPs)的 $\beta$-内酰胺类抗生素是 “特洛伊木 马” 分子策略最成功的抗生素类别. 这可能是因为 “特 洛伊木马” 策略使抗生素更容易穿过细菌外膜, 在细菌 周质中达到更高的浓度, 高浓度下抗生素更容易与周质 空间中的青霉素结合蛋白结合，发挥更佳的抗菌活性. 所以，作用于细菌细胞壁类抗生素-铁载体类偶联物的 药物研发一直是科学家和制药企业的研究热点. 首个新 型铁载体头孢菌素类抗生素头孢地尔的发现，为 “特洛 伊木马”分子抗生素的研发带来了曙光.

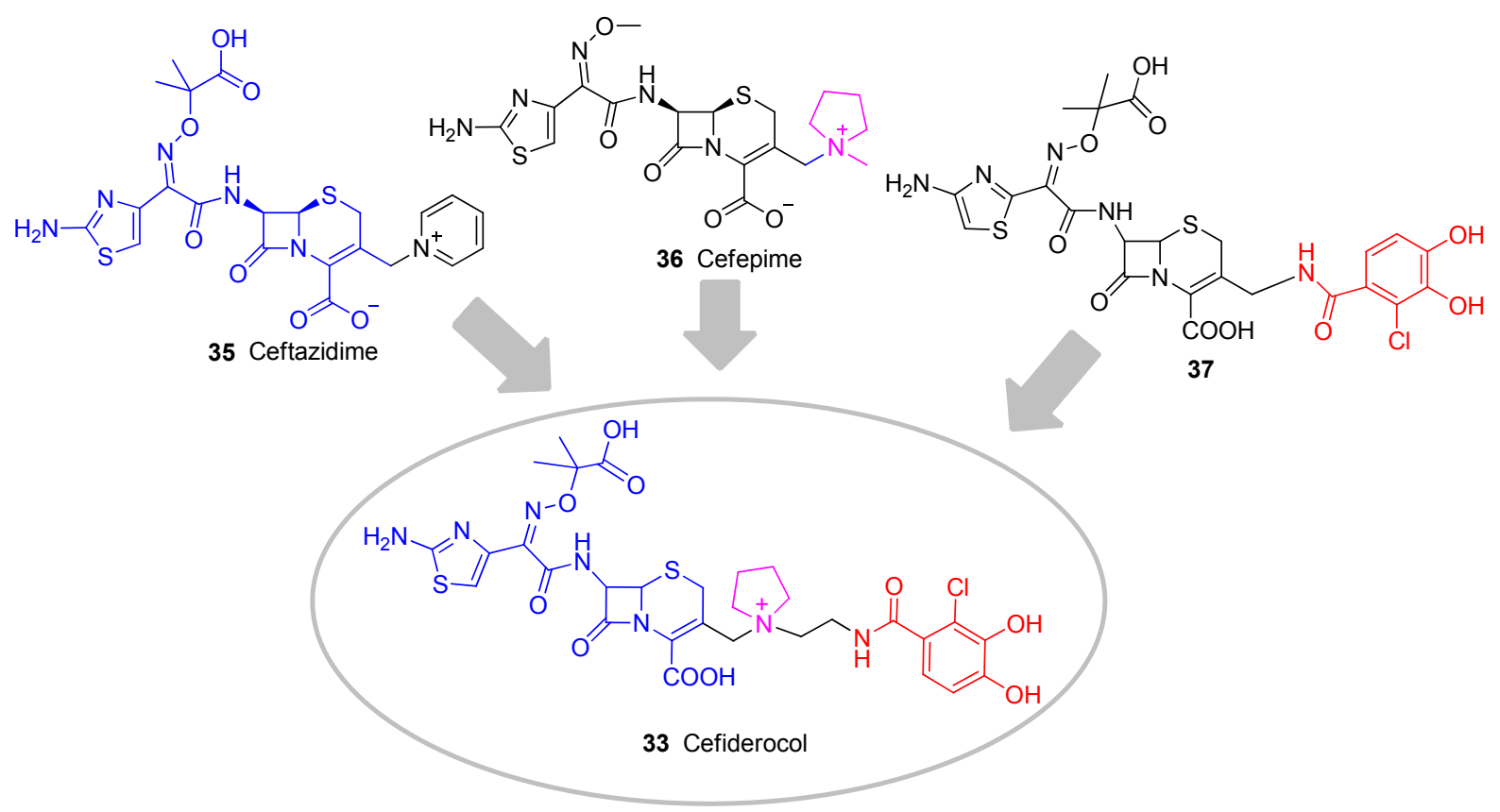

图 10 头孢地尔 $(\mathbf{3 3})$ 、头狍他啶 $\mathbf{3 5})$ 、头孢吡䏡(36) 和 $\mathbf{3 7}$ 的结构式

Figure 10 Structures of cefiderocol (33), ceftazidime (35), cefepime (36) and 37 


\section{3 作用于细菌细胞质内靶标的抗生素与铁载体 偶联物的研究}

\section{1 作用于细菌核糖体类抗生素-铁载体偶联物}

早在 1995 年, 为了确定作用于核糖体的抗生素是 否可以作为偶联物的药物部分, Miller 课题组 ${ }^{[58]}$ 率先合 成三异羟肜酸酯铁载体-红霉素胺偶联物 38 和 39(图 11). 初步活性结果表明, 这些偶联物的抗菌活性不及红 霉素胺本身抗菌活性. 1998 年, Poras 等 ${ }^{[59]}$ 随后合成了一 系列儿茶酚铁载体-螺旋霉素偶联物(40４6)(图 11)来 探究该系列偶联物的抗菌活性. 遗憾的是, 在铁离子缺 乏和铁离子充足的两种琼脂培养基条件下的活性测定 中, 也没有发现优于母体螺旋霉素抗菌活性的偶联物. 大多数作用于细菌核糖体类抗生素-铁载体偶联物的 “特洛伊木马” 策略均未取得成功, 它们的抗菌活性和 抗菌谱几乎没有优于母体抗生素. 这些大环内酯类抗生 素与铁载体偶联物的抗菌活性比原抗生素没有显著提 升, 可能是由于偶联物无法正常转运或释放到达细菌内 核糖体作用靶点所致。

由于大环内酯类抗生素与铁载体偶联物的抗菌局 限性, 我们分析作用于细菌核糖体类抗生素-铁载体偶 联物的一个限制因素, 可能是偶联物无法实现高效细菌 内膜转运, 其铁载体部分可能是影响抗生素跨过细菌内 膜的转运效率的原因; 此外, 即使部分偶联物可顺利穿 过细菌内膜, 偶联物也可能由于到达核糖体后缺乏相应 的抗生素释放机制, 从而在一定程度上降低抗生素与其 作用靶点的结合能力, 导致抗菌活性降低. 因此, 对于 此类偶联物来说, 连接基团的选择至关重要, 既要保证 偶联物在细菌外环境中不会提前水解, 又不能过于稳 定, 必须保证偶联物在细胞质内水解释放抗菌成分以发 挥抗菌作用. Schalk 课题组 ${ }^{[60]}$ 合成了噁唑烷酮类抗生素 与儿茶酚型铁载体连接得到的偶联物, 进一步探究 “特 洛伊木马” 分子抗生素策略对噁唑烷酮类抗生素的抗 菌谱的影响. 噁唑烷酮类抗生素是继磺胺和喹诺酮类抗 生素新发展起来的合成类抗菌药, 其利奈唑胺已经被批 准用于临床治疗革兰氏阳性菌感染, 特别是多重耐药的 革兰氏阳性菌感染, 都具有优秀的抗菌活性 ${ }^{[61-62]}$, 但对 铜绿假单胞菌等革兰氏阴性菌效果不佳 ${ }^{[63]}$. 初步活性 结果表明, 偶联物 47(图 12)对铜绿假单胞菌 PAO1 菌株 的 $\mathrm{MIC}$ 值为 $128 \mu \mathrm{mol} / \mathrm{L}$, 其抗菌活性优于对照的利奈唑 胺 $(\mathrm{MIC}>1024 \mu \mathrm{mol} / \mathrm{L})$. 这是一个良好的开端, 表明在 缺铁条件下, 噁唑烷酮类抗生素的 “特洛伊木马” 策略 具有可行性. 随后, 在另一项研究中, Schalk 课题组 ${ }^{[64]}$ 将噁唑烷酮类抗生素与铜绿假单胞菌的铁载体 pyochelin 结合, 得到偶联物 48(图 12) 及其类似物. 遗憾

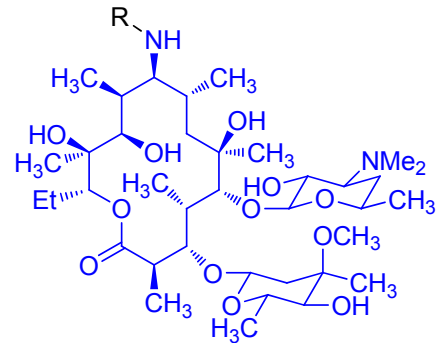

Erythromycylamine
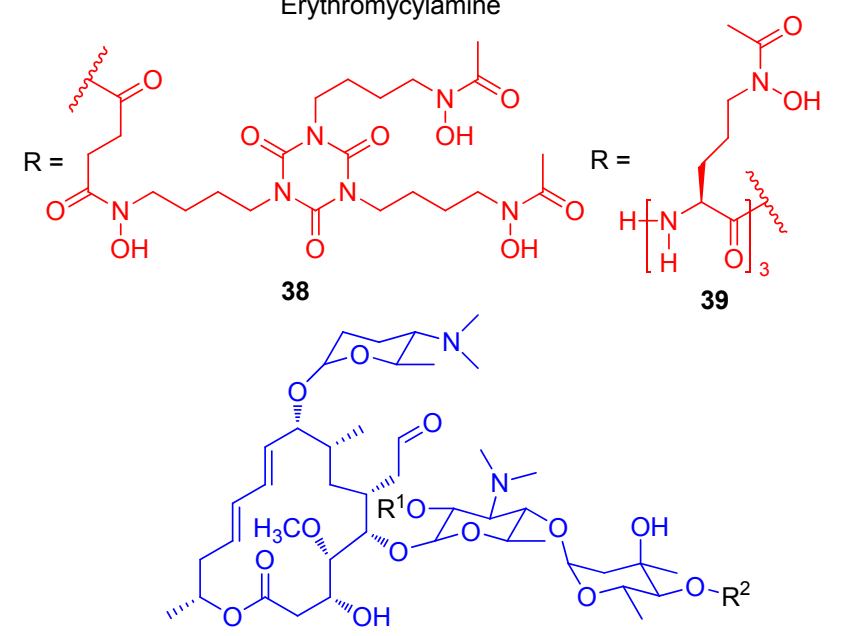

$$
\begin{aligned}
& 40 \mathrm{R}^{1}=\mathrm{Ac} ; \mathrm{R}^{2}=\mathrm{H}(\text { Spiramycin }) \\
& 41 \mathrm{R}^{1}=\mathrm{Ac} ; \mathrm{R}^{2}=\mathrm{OCO}\left(\mathrm{CH}_{2}\right)_{3} \mathrm{CONH}\left(2,3-(\mathrm{AcO})_{2} \mathrm{C}_{6} \mathrm{H}_{3}\right) \\
& 42 \mathrm{R}^{1}=\mathrm{CO}\left(3,4-(\mathrm{AcO})_{2} \mathrm{C}_{6} \mathrm{H}_{3}\right) ; \mathrm{R}^{2}=\mathrm{H} \\
& 43 \mathrm{R}^{1}=\mathrm{CO}\left(3,4-(\mathrm{HO})_{2} \mathrm{C}_{6} \mathrm{H}_{3}\right) ; \mathrm{R}^{2}=\mathrm{H} \\
& 44 \mathrm{R}^{1}=\mathrm{CO}\left(2,3-(\mathrm{HO})_{2} \mathrm{C}_{6} \mathrm{H}_{3}\right) ; \mathrm{R}^{2}=\mathrm{H} \\
& 45 \mathrm{R}^{1}=\mathrm{CO}\left(\mathrm{CH}_{2}\right)_{3} \mathrm{CONH}\left(2,3-(\mathrm{AcO})_{2} \mathrm{C}_{6} \mathrm{H}_{3}\right) ; \mathrm{R}^{2}=\mathrm{H} \\
& 46 \mathrm{R}^{1}=\mathrm{COCH}-(\mathrm{S})-\left(\mathrm{CH}_{3}\right)-\mathrm{NHCO}\left(2,3-(\mathrm{AcO})_{2} \mathrm{C}_{6} \mathrm{H}_{3}\right) ; \mathrm{R}^{2}=\mathrm{H}
\end{aligned}
$$

图 11 大环内酯类抗生素-铁载体偶联物

Figure 11 Macrolide antibiotics-siderophore conjugates

的是, 这项研究中所有的偶联物几乎没有抗菌活性. 作 者认为这可能是因为噁唑烷酮类抗生素难以从偶联物 上释放, 导致偶联物溶解度过低失活 ${ }^{[65]}$. 近期, Miller 课 题组 ${ }^{[66-67]}$ 设计并合成了一种独特的铁载体与头孢菌素 和噁唑烷酮双药偶联物 49(图 12), 其中头狍菌素充当连 接基团的主要成分, 作者设想头狍菌素作为连接基团可 以被细菌的青霉素结合蛋白或 $\beta$-内酰胺酶裂解释放噁 唑烷酮类抗生素, 以提高偶联物的抗菌活性. 偶联物 49 的抗菌活性比头狍菌素或缺乏 $\beta$-内酰胺连接的铁载体噁唑烷酮偶联物要高出 125 倍以上, 尤其是在 $\beta$-内酰胺 酶高表达的鲍曼不动杆菌株仍可保持一个较高的抗菌 活性 (MIC 值为 $6 \mu \mathrm{mol} / \mathrm{L}$ ). 进一步以鲍曼不动杆菌为参 考菌株, 通过实验验证 49 和头孢菌素-铁载体偶联物对 正常的鲍曼不动杆菌 ATCC 17978 的 MIC 均为 0.4 $\mu \mathrm{mol} / \mathrm{L}$ ，表明这两种偶联物对正常菌株的抗菌活性均归 因于头狍菌素的抗菌活性. 之后，作者通过进一步构建 质粒编码的 $\beta$-内酰胺酶(ADC-1: 一种鲍曼不动杆菌固 有的内酰胺酶, 能够水解头孢菌素类抗生素)过表达的 
鲍曼不动杆菌 ATCC 17978 菌株进行测试, 发现头孢菌 素-铁载体偶联物表现出了明显的耐药性 $(\mathrm{MIC}>50$ $\mu \mathrm{mol} / \mathrm{L})$, 而偶联物 49 仍保持良好的抗菌活性 $(\mathrm{MIC}=6$ $\mu \mathrm{mol} / \mathrm{L})$, 表明在 $\beta$-内酰胺酶高表达菌株中噁唑烷酮类 化合物主要发挥抗菌作用. Miller 课题组关于铁载体-噁 唑烷酮类偶联物的最新研究进展向我们提供了一个成 功的例子, $\beta$-内酰胺类化合物可以作为一个理想的连接 体, 将其应用于其它以胞质为靶点的铁载体-抗生素偶 联物中, 为 “特洛伊木马” 分子抗生素策略提供了新的 方向.

\section{2 作用于细菌 DNA 螺旋酶和拓扑异构酶类抗生素一 铁载体偶联物}

为了探索作用于细菌 DNA 螺旋酶和拓扑异构酶类 抗生素-铁载体偶联物作为 “特洛伊木马” 分子抗生素 策略的可行性, 2006 年, Rivault 等 ${ }^{[68]}$ 合成以螯铁蛋白为 铁载体的诺氟沙星喹诺酮类抗生素偶联物 $50 \sim 53$ (图 13). 初步抗菌活性评估发现，不稳定连接基团(亚甲基 二氧基酯)的偶联物 51 和 53 的抗菌活性与母体抗生素 诺氟沙星相比, 显示出相同的铜绿假单胞菌的抗菌活 性, 作者猜测造成这种结果是由于 51 和 53 偶联物连接

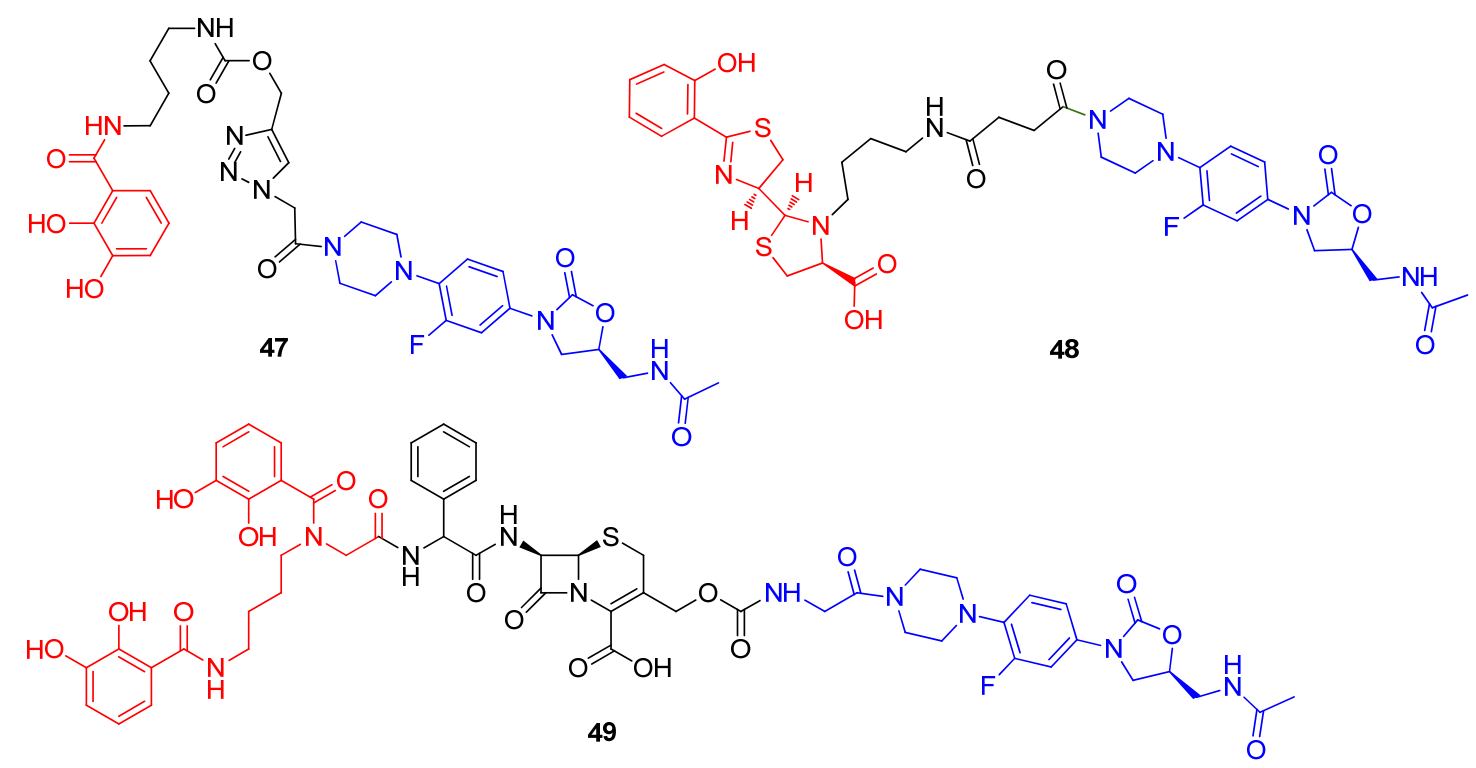

图 12 啞唑烷酮类-抗生素铁载体偶联物

Figure 12 Oxazolidinone-siderophore conjugates<smiles>CCn1cc(C(=O)O)c(=O)c2cc(F)c(N3CCN(C(=O)OCOC(=O)CCC(=O)NCC#Cc4ccc(O)c(C5=NC(C6SCC(C(=O)O)N6C)CS5)c4)CC3)cc21</smiles><smiles>CCn1cc(C(=O)O)c(=O)c2cc(F)c(N3CCN(C(=O)CCC(=O)NCCCc4ccc(O)c(C5=NC(C6SCC(C(=O)O)N6C)CS5)c4)CC3)cc21</smiles>

图 13 诺氟沙星一铁载体类偶联物

Figure 13 Norfloxacin-siderophore conjugates 
基团不稳定, 未到达细胞质之前就被非特异性酯酶部分 水解, 而未被水解的部分偶联物进入细胞内并释放游离 的诺氟沙星, 从而发挥抗菌活性 ${ }^{[69]}$. 遗憾的是, 稳定的 连接基团(酰胺键)偶联物 $\mathbf{5 0}$ 和 $\mathbf{5 2}$, 对细菌生长曲线没有 明显的抑制作用. 对于偶联物 50、52 的失活, 作者猜测 偶联物可以借助苂光嗜铁素(pyoverdine) 被细菌受体识 别 ${ }^{[70]}$, 转运进入细菌细胞膜, 但由于连接基团太过稳 定, 使得抗生素难以释放, 造成偶联物 50、52 失活. 这 说明铁载体-抗生素偶联物中, 连接基团可能是喹诺酮 类偶联物发挥抗菌作用的关键因素, 当连接基团不易水 解时, 诺氟沙星可能无法从偶联物中释放作用于细胞质 中的 DNA 螺旋酶, 或者偶联物中铁载体部分在空间上 阻碍了诺氟沙星与 DNA 螺旋酶的相互作用, 导致抗菌 活性下降.

2013 年, Wencewicz 等 ${ }^{[71]}$ 合成了三异羟肜酸酯铁载 体与环丙沙星连接的偶联物 54(图 14), 初步活性数据显 示, 54 对革兰氏阳性菌金黄色葡萄球菌(MIC 值为 1 $\mu \mathrm{mol} / \mathrm{L})$ 具有与环丙沙星 $(\mathrm{MIC}$ 值为 $0.5 \mu \mathrm{mol} / \mathrm{L}$ )等同的抗 菌活性，但对革兰氏阴性菌无效。这说明完整的三差肜 酸铁载体骨架参与了细菌膜转运, 这种类型的转运铁载 体对具有羟肜酸铁载体转运蛋白的革兰氏阳性细菌具 有选择性. 2014 年, Fardeau 等 ${ }^{[72-73]}$ 合成了三儿茶酚基团 作为铁载体, 可水解的酯键作为连接基团, 环丙沙星作 为抗生素的偶联物 $55 \sim 58$ (图 14), 探究该系列偶联物的 抗菌活性. 初步活性结果表明, 偶联物 55 和 57 对铜绿 假单胞菌 DSM 1117 呈现中等的抗菌活性(MIC 值分别 为 8 和 $64 \mu \mathrm{g} / \mathrm{mL}$ ), 而 58 在 $128 \mu \mathrm{g} / \mathrm{mL}$ 依然没有呈现抗 菌活性, 所有偶联物的抗菌活性均低于母体抗生素环丙 沙星. 这可能是因为偶联物在水性介质中的溶解度低或 在细胞质中连接基团酯键未发生水解或部分水解导致 抗菌成分未得到完全释放.

综上，我们可以发现 “特洛伊木马” 分子以喹诺酮 类抗生素来探究时，连接基团对抗菌活性有至关重要的 影响, 为了更深入探究铁载体-抗生素偶联物中连接基<smiles>O=C(CCC(=O)N(O)CCCCCO)NCCCCCN(O)C(=O)CCC(=O)NCCCCCN(O)C(=O)CCC(=O)N1CCN(c2cc3c(cc2F)c(=O)c(C(=O)O)cn3C2CC2)CC1</smiles><smiles>O=C(O)c1cn(C2CC2)c2cc(N3CCN(CCCC(=O)N4CCN(c5cc6c(cc5F)c(=O)c(C(=O)O)cn6C5CC5)CC4)CC3)c(F)cc2c1=O</smiles>

图 14 环丙沙星一铁载体类偶联物

Figure 14 Ciprofloxacin-siderophore conjugates 
才对抗菌活性的影响. 2012 年, $\mathrm{Ji}$ 等 ${ }^{[74]}$ 设计并合成了以 邻羟基肉桂酸衍生物为连接基团的环丙沙星偶联物 59 (图 15). 邻羟基肉桂酸衍生物作为连接基团, 易被细菌 和真菌分泌的酯酶或磷酸酶内酯化, 迅速形成氢化香豆 素, 并伴随抗生素的释放. 初步抗菌活性评估 $\mathbf{5 9}$ 对革兰 氏阳性菌株(枯草芽孢杆菌、金黄色葡萄球菌)以及铜绿 假单胞菌呈现中等抗菌活性( $\mathrm{MIC}_{90}$ 为 $\left.1 \sim 32 \mu \mathrm{g} / \mathrm{mL}\right)$, 而 对粪肠球菌无抗菌活性. 遗憾的是, 59 抗菌活性低于母 体抗生素环丙沙星, 可能是酯酶水解导致抗生素释放不 完全 ${ }^{[75]}$. 2015 年, Zheng 等 ${ }^{[76]}$ 设计并合成了带有不稳定 (酰氧基)烷基酯为连接基团与肠杆菌素铁载体和环丙沙 星偶联物 60(图 15), 进一步探究连接基团对抗菌活性的 影响. 先前的研究表明, (酰氧基)烷基酯连接基对空间 环境敏感 ${ }^{[77]}$, 通过调节(酰氧基)烷基酯附近的取代基, 增大位阻, 可增强偶联物的稳定性, 避免药物的过早释 放. 2018 年, Neumann 等 ${ }^{[78]}$ 报道了以烷基链为连接基团, 肠杆菌素铁载体与环丙沙星偶联得到的偶联物 61(图 15), 探究了烷基类连接基团对抗菌活性的影响, 并评 估了偶联物药物释放作用机制. 烷基连接的偶联物 61 对两种大肠杆菌 UTI89 和大肠杆菌 CFT073 都具有很高 的抗菌活性, 其 MIC 值与环丙沙星(MIC 值为 $0.1 \sim 1$ $\mu \mathrm{mol} / \mathrm{L})$ 接近. 已有研究 ${ }^{[79]}$ 和当前研究的结果表明, 连 接基团可能会阻止偶联物的跨膜转运和药物释放. 随后<smiles>CCN(C)c1cc2c(cc1F)CC(C(=O)O)=CN2C1CC1</smiles>

59<smiles>CCOCC(NC(=O)c1cc(C(=O)NCCCCCC(=O)N2CCN(c3cc4c(cc3F)c(=O)c(C(=O)O)cn4C3CC3)CC2)cc(O)c1O)C(=O)OC</smiles>

61<smiles>O=C(NC(CCC(=O)[C@H](CO)NC(=O)c1cccc(O)c1O)C(=O)[O-])c1cccc(O)c1O</smiles>

图 15 环丙沙星-铁载体类偶联物

Figure 15 Ciprofloxacin-siderophore conjugates
Neumann 等 $^{[80]}$ 合成了不稳定二硫键作为连接基团的偶 联物 62(图 15), 探讨了连接基团的稳定性对偶联物抗菌 活性的影响, 遗憾的是, 62 只对部分大肠杆菌株有生长 抑制作用. 作者猜测 62 可能在未进入细菌细胞膜时提 前还原裂解释放抗生素，所以二硫键不能广泛应用于铁 载体一抗生素偶联物的设计中. 偶联物主要是通过连接 基团使铁载体与喹诺酮类抗生素偶联，我们发现连接基 团的选择对喹诺酮类偶联物抗菌活性的提升至关重要, 大部分可水解的连接基团，由于其不稳定性容易导致抗 生素过早释放, 而不可水解的连接基团相比可水解的连 接基团, 可使偶联物在体外和转运过程中更加稳定, 然 后在细菌内使偶联物释放, 提高偶联物的抗菌活性. 但 过于稳定的连接基团, 又增加了抗生素释放的难度, 比 如, 偶联物 62 与母体抗生素相比, 抗菌活性并没有提 高.

喹诺酮类抗生素作为 “特洛伊木马” 分子的抗生 素时, 偶联物的抗菌活性大都没有提高, 主要原因可能 有以下几种: (1)偶联物自身溶解性的问题; (2)铁载体结 构较大, 阻碍了偶联物转运至细胞质与喹诺酮类抗生素 的作用靶标结合; (3)铁载体与抗生素之间的连接基团不 可水解, 不能释放抗生素; (4)连接基团具有非特异性水 解的特性, 可能还未到达药物作用靶标过早释放等.<smiles>CC(OC(=O)C(C)NC(=O)c1cc(O)c(O)c(C(=O)NC(COC(=O)C(COC(=O)C(COC(=O)[C@H](C)NC(=O)c2cccc(O)c2O)NC(=O)c2cccc(O)c2O)NC(=O)c2cccc(O)c2O)C(=O)O)c1)OC(=O)N1CCN(c2cc3c(cc2F)c(=O)c(C(=O)O)cn3C2CC2)CC1</smiles>

60 $\mathrm{OH}$ 


\section{4 作用于细菌细胞膜类抗生素-铁载体偶联物 的研究}

\section{1 达托霉素-铁载体偶联物}

达托霉素是由玫瑰孢链霉菌(Streptomyces roseosporus)发酵得到的含有十个碳脂肪侧链的环脂肽. 2003 年, 达托霉素(Cubicin)获得美国食品与药物管理局 (FDA)批准, 用于治疗革兰氏阳性菌引起的并发性皮肤 感染, 并在美国等多个国家上市. 达托霉素可以与细菌
细胞膜结合并破坏其功能，使内容物外泄而发挥杀菌的 作用 ${ }^{[81]}$. 但由于达托霉素分子量大，具有阴电性的特 点, 导致其无法穿过革兰氏阴性菌外膜. 因此, 临床上 达托霉素主要用于治疗革兰氏阳性菌引起的感染. 2017 年, Gosh 等 ${ }^{[82]}$ 将天然鲍曼不动杆菌(Acinetobacter baumannii)的 fimsbactin A 铁载体进行改造, 人工合成了结 构中包含两个儿茶酚和一个异羟肜酸酯的 fimsbactin A 铁载体类似物，再与达托霉素通过酰胺键连接得到偶联 物 63(图 16). 初步活性研究发现，偶联物 63 对革兰氏阳

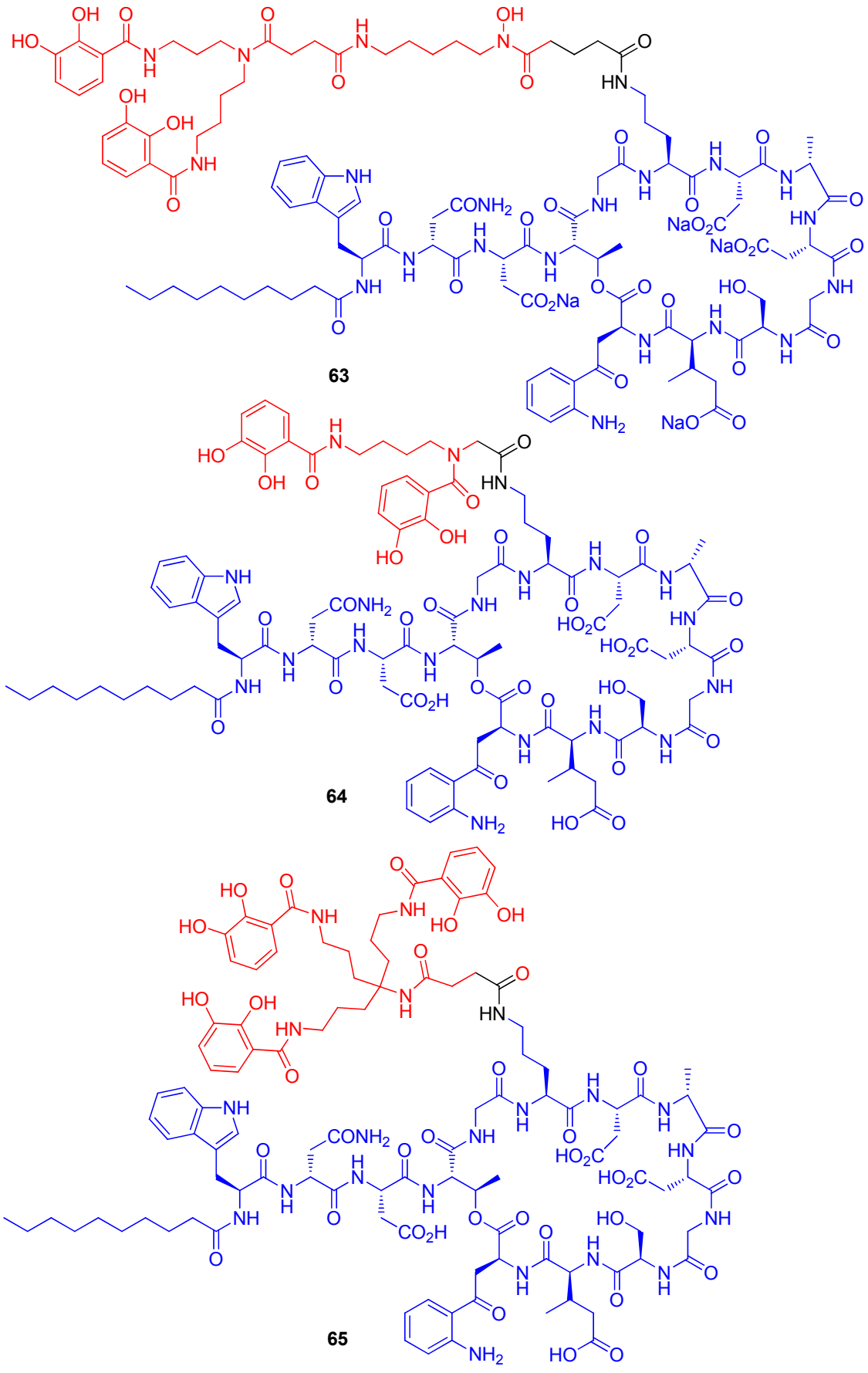

图 16 达托霉素-铁载体类偶联物

Figure 16 Daptomycin-siderophore conjugates 
性菌金黄色葡萄球菌菌株的 MIC 大约为 $6 \sim 12.5$ $\mu \mathrm{mol} / \mathrm{L}$ ，其抗菌活性低于达托霉素 $(\mathrm{MIC}=0.05 \sim 0.4$ $\mu \mathrm{mol} / \mathrm{L}$ ). 但是, 与对革兰氏阴性菌生长无抑制作用的 达托霉素相比, 63 对鲍曼不动杆菌及其耐药菌株的 MIC 为 $0.4 \sim 0.8 \mu \mathrm{mol} / \mathrm{L}$, 表明人工合成的 fimsbactin $\mathrm{A}$ 铁载 体类似物可以被革兰氏阴性菌外膜的转运蛋白识别, 偶 联物可以通过主动转运进入细胞周质中, 与细菌细胞膜 结合发挥抗菌活性. 达托霉素作用靶点位于细胞膜而不 在细胞质中, 所以连接部分是否水解并不影响偶联物的 抗菌活性. 2018 年, Miller 课题组 ${ }^{[83]}$ 对偶联物 63(图 16) 进行结构改造, 将 fimsbactin A 铁载体类似物替换为双 儿茶酚类(bis-catechol)和三儿茶酚类(tri-catechol)结构的 铁载体, 分别合成偶联物 64 和 65 (图 16). 初步活性检测 发现, 偶联物 64 和 65 对鲍曼不动杆菌及其耐药菌株抗 菌活性均弱于偶联物 63 , 可能是因为人工改造的铁载 体 fimsbactin A 类似物与人工合成儿茶酚类铁载体相比, 更易于被鲍曼不动杆菌细胞外膜上的转运蛋白识别, 使 得偶联物更高效地被转运到细菌周质内.

此外, 三种达托霉素与铁载体连接得到的偶联物对 鲍曼不动杆菌外的其它革兰氏阴性菌大肠杆菌以及铜 绿假单胞菌均未显现出抗菌活性, 这与达托霉素抗菌作 用靶点在大肠杆菌以及铜绿假单胞菌分布较少有关 ${ }^{[84]}$. 综上, 偶联物 63 能够通过铁载体介导的铁离子吸收系 统, 跨过鲍曼不动杆菌的外膜到达细胞膜发挥抗菌活 性, 是 “特洛伊木马” 策略拓宽达托霉素抗菌谱成功的 典型例子, 为后续抗菌药物研发提供了全新的可能.

\section{2 抗菌肽 gallidermin-铁载体偶联物}

Lantibiotic 是一类由革兰氏阳性菌产生, 结构中含 有羊毛硫氨酸(lanthionine), 作用于其它革兰氏阳性菌 的抗菌肽. 根据化学结构和抗菌活性, lantibiotic 类抗菌 肽分为 $\mathrm{A}$ 型和 $\mathrm{B}$ 型. $\mathrm{A}$ 型 lantibiotic 类抗菌肽是正电荷、
结构较为延伸的肽. B 型是一些较少量正电荷、结构为 球形小分子的肽 ${ }^{[85]}$. Lantibiotic 类抗菌肽与细胞膜结合 后，以表面结合的方式插入到细胞膜的双分子层，进一 步导致细胞渗漏和死亡 ${ }^{[86]}$. 但是, lantibbotic 类抗菌肽缺 乏穿透革兰氏阴性菌细胞外膜的能力，限制了此类抗菌 肽的使用范围. 为了改善这类抗菌肽的缺陷, 2011 年 Yoganathan 等 ${ }^{[87]}$ 对 gallidermin (A 型 lantibiotic 类抗菌肽) 进行结构改造, 在位于 13 号位的赖氨酸上引入人工合 成的 agrobactin 铁载体类似物, 连接部分为方酸二甲胺 (diamino squarate)得到偶联物 66(图 17). 初步活性数据 显示, 与母体抗菌肽相比, 66 保留了对阳性菌的抗菌活 性，但没有产生革兰氏阴性菌的抗菌活性. 有趣的是, 偶联物 66 对铜绿假单胞菌的生长有轻微的促进作用. 推测这种反常现象是偶联物为细菌提供了少量的铁离 子导致的, 说明铁载体可以促使偶联物跨过阴性菌外 膜. 但是，可能由于 lantibiotic 类抗菌肽分子量过大，导 致穿过外膜的抗菌肽的分子数极少. 偶联物在较低浓度 下无法发挥抗菌活性，其螯合的可溶性铁离子被交换到 细菌天然铁载体中, 为细菌生长提供了少量铁离子, 从 而轻微促进了铜绿假单胞菌的生长.

综上，达托霉素-铁载体偶联物的成功验证了 “特 洛伊木马” 分子抗生素策略, 可以提升作用于细菌细胞 膜的抗生素的抗菌活性, 并扩大已有抗生素的抗菌谱, 为后续扩大抗菌谱的相关抗菌药物研发提供了全新的 策略和方向.

\section{5 其它类铁载体偶联物的研究}

\section{1 三氯生类抗生素-铁载体偶联物}

2009 年, Wencewicz 等 ${ }^{[88}$ 报道了以三氯生为抗生素, 去铁胺类似物为铁载体, 酯键作为连接片段的偶联物 67(图 18). 三氯生是一种广谱的抗菌药物, 其作用靶标

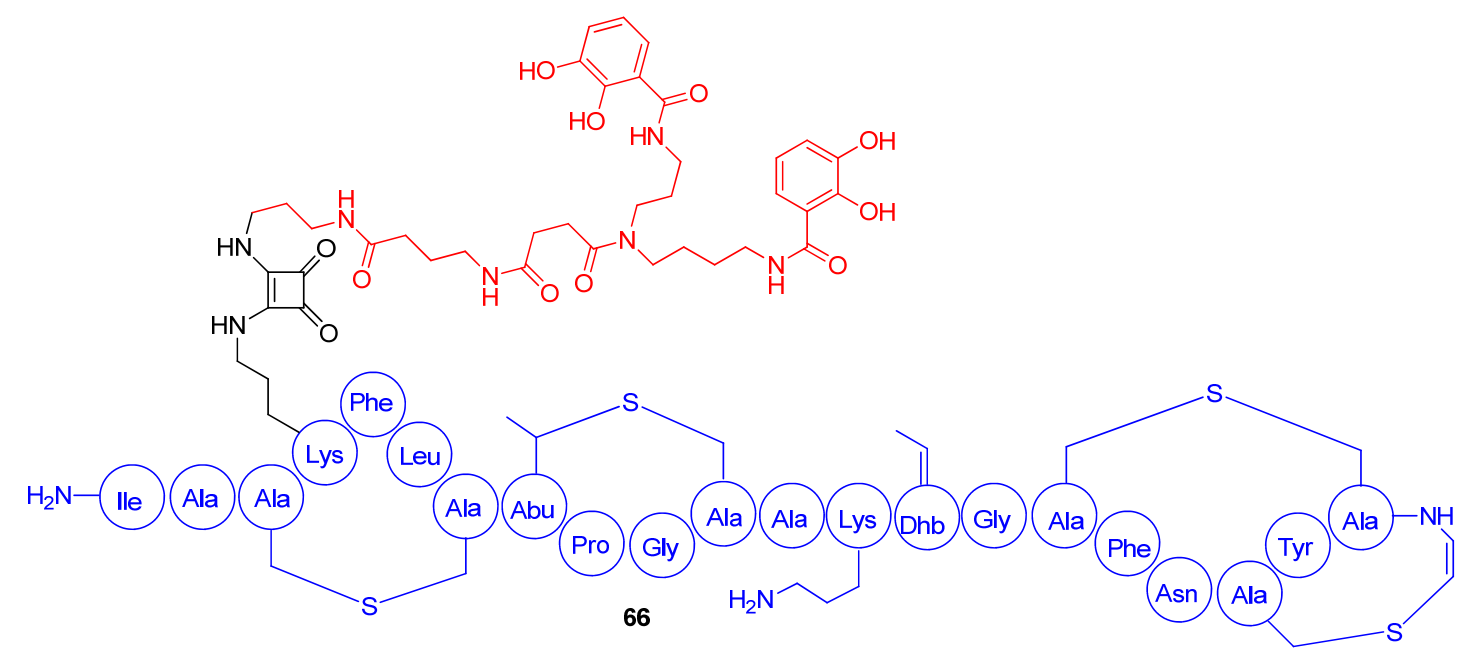

图 17 Lantibiotic-铁载体类偶联物

Figure 17 Lantibiotic-siderophore conjugates 


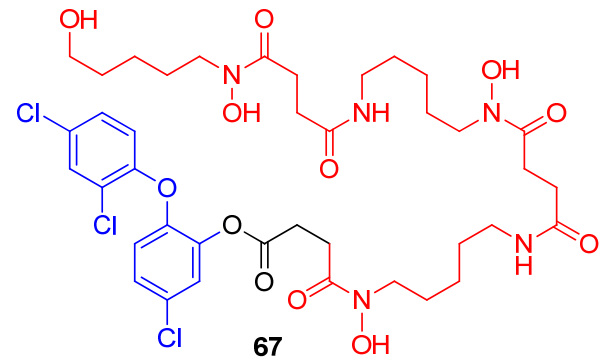<smiles>O=C(CCC(=O)N(O)CCCCCO)NCCCCCN(O)C(=O)CCC(=O)NCCCCN(O)C(=O)CCC(=O)NC(C(=O)N[C@H]1C(=O)N2C(C(=O)O)=C(Cl)CC[C@@H]12)c1ccccc1</smiles>

图 18 三氯生-铁载体偶联物

Figure 18 Triclosan-siderophore conjugates

为烯脂酰载体蛋白还原酶(FabI). FabI 在细菌体内起到 重要作用, 它是脂肪酸合成途径中的限速酶 ${ }^{[89]}$. 三氯生 通过与 FabI 以及烟酰胺辅因子形成稳定的三元配合物 来影响 FabI 发挥作用, 抑制细菌体内的脂肪酸的合成 [90]. 偶联物的生物活性实验表明, 67 对革兰氏阳性菌金 黄色葡萄球菌(Staphylococcus aureus)、革兰氏阴性菌大 肠杆菌 (E. coli)、铜绿假单胞菌(Pseudomonas aeruginosa) 的生长都具有抑制作用, 对大肠杆菌菌株 E. coli DC2 的 $\mathrm{MIC}$ 值小于 $0.1 \mu \mathrm{mol} / \mathrm{L}$. 偶联物 67 的抗菌活性与母体三 氯生的作用效果相似. 其机制研究表明, 所用的大肠杆 菌菌株均无法利用去铁胺来摄取培养基中的铁离子，表 明偶联物中铁载体并没有发挥将三氯生转运到细菌胞 内的作用. 因此, 三氯生在细菌胞外的提前释放可能是 偶联物保持抗菌活性的根本原因.

Miller 课题组 ${ }^{[71,88]}$ 受沙霉素独特的作用机制启发, 设计了一系列铁载体一抗生素偶联物, 它们分别是通过 特异性模拟沙霉素三异羟肜酸铁载体与不同作用机制 的抗生素氯碳头孢 68 (以细菌细胞壁为靶标的抗生素)、 环丙沙星 54(以细菌 DNA 螺旋酶和拓扑异构酶为靶标 的抗生素) 和三氯生 67 (以烯脂酰载体蛋白还原酶为靶标 的抗生素)结合形成的偶联物. 他们发现, 在几乎所有 测试的菌株中, 偶联物 54 和 68 与它们的母体抗生素氯 碳头孢和环丙沙星相比, 抗菌活性降低, 这可能是由于
它们缺乏相应的抗生素释放机制所致. 进一步分析发 现，与酰胺作为连接部分的偶联物 54 和 68 相比，以酚 酯作为连接部分的偶联物 67 具有更高的水解不稳定性, 极有可能在胞外环境中提前释放游离三氯生，发挥其抗 菌作用. 综上, 这说明连接部分对偶联物转运进入细菌 胞内发挥抗菌活性起重要作用，连接基团需具有某些特 定条件下分解的特性，以保证偶联物可以进入细菌细 胞，并在细胞内使药物释放.

\section{2 铁载体-抗真菌化合物 desketoneoenactin 偶联物}

2005 年 Miller 课题组 ${ }^{[91]}$ 报道了以具有抑制真菌生 长的化合物 desketoneoenactin (DE) 作为药效基团, 合成 了三个连接不同铁载体的偶联物 69，70 和 71(图 19). Desketoneoenactin 具体作用机制并不明确，推测可能是 因为分子结构中含有疏水基团和亲水基团，化合物具有 两性，可以插入到细胞膜中，通过破坏细胞膜的完整性 来杀死真菌 ${ }^{[92]}$. 偶联物 69 中铁载体部分为高铁色素铁 载体类似物(ferrichrome, $\mathrm{FCH}$ ), 偶联物 70 中铁载体部 分为鸟氨酸铁载体类似物 (ornithine-based trihydroxamate, ORNI), 偶联物 71 中的铁载体部分为异氰脲酸酯 类铁载体类似物(isocyanurate-based hydroxamate, ISO). 初步活性探究发现, 缺铁条件下偶联物 69, 70 和 71 对白 色念珠菌的 MIC 值依次为 3.1, 12.5 和 $25 \mu \mathrm{mol} / \mathrm{L}$. 遗憾<smiles>CCCCCCCCCCCC(=O)CCN(O)C(=O)[C@H](CO)NC(=O)C(N)CCCN(O)C(C)=O</smiles>

69<smiles>CCCCCCCCCCCC(=O)CCN(O)C(=O)[C@H](CO)NC(=O)C(CN(O)C(C)=O)NC(=O)CNC(=O)C(CCCN(O)C(C)=O)NC(=O)C(N)CCCN(O)C(C)=O</smiles><smiles>[Y]ON(CCCCn1c(=O)n(CCCCN(O)C(C)=O)c(=O)n(CCCCN(O)C(C)=O)c1=O)C(=O)CCC(=O)N[C@@H](CO)C(=O)N(O)CCC(=O)CCCCCCCCCCCC</smiles>

图 19 Desketoneoenactin-铁载体类偶联物

Figure 19 Desketoneoenactin-siderophore conjugates 
的是, 该系列偶联物的抗菌活性均低于 desketoneoenactin (MIC 值为 $0.2 \mu \mathrm{mol} / \mathrm{L}$ ). 进一步机制探究中发现 白色念珠菌更易识别高铁色素铁载体, 结构中含有高铁 色素铁载体类似物的偶联物 69 更易于被真菌摄取, 抗 菌活性也优于其它两种偶联物. 不同真菌菌株对铁载体 可能存在特异选择性, 铁载体直接影响到偶联物的抗菌 活性, 所以根据不同真菌菌株, 选取不同种类铁载体是 真菌类抗生素偶联物设计中必需考虑的关键因素.

综上, 连接部分的稳定性对偶联物发挥抗菌活性起 重要作用. 如果连接部分在胞外水解, 与母体抗生素相 比, 偶联物的抗菌活性会下降甚至消失. 所以, 连接部 分的选择是 “特洛伊木马” 策略抗生素成功的关键因素. 由于菌株对不同类型铁载体的摄取具有特异性, 因此, 科研工作者在设计偶联物时也应该评价目标菌株对不 同种类铁载体的摄取能力, 䇻选出易被目标菌株利用的 铁载体作为偶联物中的药物递送部分.

\section{6 总结与展望}

“特洛伊木马” 分子策略作为新作用模式的抗生 素在实验和临床方面都取得了一定的进展, 并具有巨大 的研发潜能与应用前景. “特洛伊木马” 分子偶联物是 由三个部分组成: 铁载体(结构式中红色部分)、连接基 团 (结构式中黑色部分)和抗生素 (结构式中蓝色部

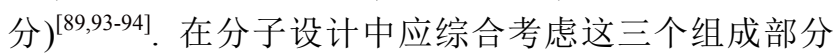
中的每一组分对抗菌效果的影响, 进行系统选择和优 化, 以便在膜渗透和抗生素释放方面产生最佳抗菌活 性. 大量研究显示, 这些部分的选择和优化具有一定的 困难性, 这也是迄今为止只有极少数成功的 “特洛伊木 马” 抗生素出现的原因. 铁载体-抗生素偶联物发展的 第一个难点是寻找适用于 “特洛伊木马” 策略的抗生 素, 大多数作用于细菌细胞质内靶标的抗生素偶联的 “特洛伊木马” 偶联物 $(\mathbf{4 1}, 47)$ 通常显示出低于母体抗 生素的抗菌活性. 这种限制主要存在两种可能, 第一种 是由于抗生素偶联的铁载体和细菌自身分泌的铁载体 与细菌膜转运蛋白的转运选择性差异而导致携带抗生 素的铁载体很难到达细胞质中的作用靶点 ${ }^{[22,29,59-63,95]}$; 另一种可能是因为外周胞质中细菌天然铁载体释放铁 离子阻挡偶联物进入细菌发挥抗菌活性. 所以, 对于革 兰氏阴性菌, 大多数铁载体-抗生素偶联物只能结合到 位于外周胞质的青霉素结合蛋白, 这也解释了为什么迄 今为止大多数成功的 “特洛伊木马” 策略都选择作用 在细菌细胞壁和细胞膜等细菌外周胞质靶标的抗生素, $\beta$-内酰胺抗生素 $(47,33,49)$ 就是最好的例子. 铁载体-抗 生素偶联物发展的另一个难点是寻找适用于 “特洛伊 木马” 策略的连接基团. 理想的连接基团不仅能在细胞
外部条件下稳定, 也能在铁吸收系统的主动转运过程中 稳定, 并在细菌内使铁载体-抗生素偶联物裂解释放抗 生素. 但迄今为止发现的连接基团还远未达到理想状 态. 大部分可水解的连接基团, 由于其不稳定性容易导 致抗生素过早释放. 与不可水解的连接基团相比, 可水 解的连接基团可使偶联物在体外和转运过程中更加稳 定, 然后在细菌内使偶联物释放抗生素, 提高偶联物的 抗菌活性. 但过于稳定的连接基团又增加了抗生素释放 的难度, 还需要进一步的优化改造 $(\mathbf{5 5} \sim 57)^{[60-65,71-76]}$. Miller 课题组关于铁载体-噁唑烷酮类偶联物的最新研 究进展向我们提供了一个成功的例子, $\beta$-内酰胺类化合 物 $(47,49)$ 也可以作为一个理想的连接基团, 将其应用 于其它以胞质为靶点的铁载体-抗生素偶联物中. 在 “特洛伊木马” 策略中还要考虑的铁载体-抗生素偶联 物的第三部分是铁载体. 迄今为止，已经描述了大量的 铁载体-抗生素偶联物, 但与天然铁载体相比, 人工合 成的儿茶酚或异羟肟酸酯类铁载体是最有效的铁载体 类似物 $(20,22 \sim 24)^{[38-42,88,93,96]}$. 混合型铁载体相较于单 一型铁载体展现出更优的药物递送能力, 更有助于偶联 物发挥抗菌作用. 所以，优化 “特洛伊木马” 分子偶联 物中抗生素、连接基团和铁载体三个组成部分, 可能是 未来铁载体类抗生素研究的主要方向.

铁载体-抗生素药物设计策略为新型作用机制类抗 生素药物研发提供了新的发展方向, 与母体抗生素相 比，“特洛伊木马” 策略偶联物的选择性和抗菌活性的 提高还可以降低抗生素耐药性的风险. 迄今为止，最成 功的铁载体-抗生素是作用于细菌周质靶标的抗生素 $\beta$ 内酰胺类的头孢地尔, 该铁载体偶联抗生素的发现会继 续刺激对铁载体-抗生素偶联物的进一步研究. 作者认 为, $\beta$-内酰胺-铁载体偶联物虽然一直是科学家和制药 企业的药物研发热门, 但非 $\beta$-内酰胺抗生素-铁载体偶 联物似乎仍然是学术上的追求. 铁载体-抗生素偶联物 的潜在化学药物研发空间巨大，相信不久的将来，其将 会是未来新型作用模式抗生素的重要来源.

\section{References}

[1] Zaman, S. B.; Hussain, M. A.; Nye, R. Mehta, V.; Mamun, K. T.; Hossain, N. Cureus 2017, 9, 1403.

[2] Brown, E. D.; Wright, G. D. Nature 2016, 529, 336.

[3] Blair, J. M.; Webber, M. A.; Baylay, A. J.; Ogbolu, D. O.; Piddock, L. J. Nat. Rev. Microbiol. 2015, 13, 42.

[4] Rossiter, S. E.; Fletcher, M. H.; Wuest, W. M. Chem. Rev. 2017, $117,2415$.

[5] Lewis, K. Nat. Rev. Drug Discovery 2013, 12, 371.

[6] Abouelhassan, Y.; Garrison, A, T.; Yang, H.; Chavez-Riveros, A.; Burch, G. M.; Huigens, R. W. I. J. Med. Chem. 2019, 6, 7618.

[7] Soares, M. P.; Weiss, G. EMBO Rep. 2015, 16, 1482.

[8] Hider, R. C.; Kong, X. Nat. Prod. Rep. 2010, 27, 637.

[9] Gorska, A.; Sloderbach, A.; Marszall, M. P. Trends Pharmacol. Sci. 2014, 35, 442 . 
[10] Page, M. G. Ann. N. Y. Acad. Sci. 2013, 1277, 115.

[11] Ferguson, A. D.; Braun, V.; Fiedler, H. P.; Coulton, J. W.; Diederichs, K.; Welte, W. Protein Sci. 2000, 9, 956.

[12] Clarke, T. E.; Braun, V.; Winkelmann, G.; TariL, W.; Vogel, H. J. J. Biol. Chem. 2002, 277, 13966.

[13] Braun, V.; Pramanik, A.; Gwinner, T.; Koberle, M.; Bohn, E. Biometals 2009, 22, 3.

[14] Juarez-Hernandez, R. E.; Miller, P. A.; Miller, M. J. ACS Med. Chem. Lett. 2012, 3, 799.

[15] Vertesy, L.; Aretz, W.; Fehlhaber, H. W.; Kogler H. Helv. Chim. Aata 1995, 78, 46

[16] Roosenberg, J. M.; Miller, M. J. J. Org. Chem. 2000, 65, 4833

[17] Braun, V. K.; Günthner, H.; Zimmermann, L. J. Bacteriol. 1983, 156, 308 .

[18] Saha, M.; Sarkar, S.; Sarkar, B.; Sharma, B. K.; Bhattacharjee, S.; Tribedi, P. Environ. Sci. Pollut. Res. Int. 2016, 23, 3984.

[19] Bilitewski, U.; Blodgett, J. Duhme-Klair, A.K.; Dallavalle, S. Laschat, S.; Routledge, A.; Schobert, R. Angew. Chem., Int. Ed. 2017, 56, 14360.

[20] Abouelhassan, Y.; Garrison, A. T.; Yang, H.; Chavez-Riveros, A.; Burch, G. M.; Huigens, R. R. J. Med. Chem. 2019, 62, 7618.

[21] Madsen, J. L.; Johnstone, T. C.; Nolan, E. M. J. Am. Chem. Soc. 2015, 137, 9117.

[22] Ji, C.; Miller, P. A.; Miller, M. J. J. Am. Chem. Soc. 2012, 134, 9898.

[23] Mislin, G. L.; Schalk, I. J. Metallomics 2014, 6, 408.

[24] Muller, G.; Barclay, S. J.; Raymond, K. N. J. Biol. Chem. 1985, 260, 13916.

[25] Sayer, J. M.; Emery, T. F. Biochemistry 1968, 7, 184.

[26] Krewulak, K. D.; Vogel, H. J. Biochim. Biophys. Acta 2008, 1778, 1781.

[27] Miethke, M.; Marahiel, M. A. Microbiol. Mol. Biol. Rev. 2007, 71, 413.

[28] Winkelmann, G. Biometals 2007, 20, 379.

[29] Mollmann, U.; Heinisch, L.; Bauernfeind, A.; Kohler, T.; AnkelFuchs, D. Biometals 2009, 22, 615.

[30] Ballouche, M.; Cornelis, P.; Baysse, C. Recent Pat. Anti-Infect. Drug Discovery 2009, 4, 190.

[31] Murphy-Benenato, K. E.; Bhagunde, P. R.; Chen, A; Davis, H. E.; Durand-Reville, T. F.; Ehmann, D. E. J. Med. Chem. 2015, 58, 2159.

[32] Dolence, E. K.; Minnick, A. A.; Miller, M. J. J. Med. Chem. 1990, 33,461 .

[33] Mckee, J. A.; Sharma, S. K.; Miller, M. J. Bioconjugate Chem. 1991, 2, 281.

[34] Ramurthy, S.; Miller, M. J. J. Org. Chem. 1996, 61, 4120.

[35] Minnick, A. A.; Mckee, J. A.; Dolence, E. K.; Miller, M. J. Antimicrob. Agents Chem. 1992, 36, 840.

[36] Ghosh, A.; Ghosh, M.; Niu, C.; Malouin, F.; Moellmann, U.; Miller, M. J. Chem. Biol. 1996, 3, 1011.

[37] Ghosh, M.; Miller, M. J. Bioorg. Med. Chem. 1996, 4, 43.

[38] Kinzel, O.; Tappe, R.; Gerus, I.; Budzikiewicz, H. J. Antibiot. 1998, $51,499$.

[39] Heinisch, L.; Wittmann, S.; Stoiber, T.; Berg, A.; Ankel-Fuchs, D.; Mollmann, U. J. Med. Chem. 2002, 45, 3032.

[40] Heinisch, L.; Wittmann,S.; Stoiber, T.; Scherlitz-Hofmann, I.; Ankel-Fuchs, D.; Mollmann, U. Arzneim. Forsch. 2003, 53, 188.

[41] Ji, C.; Miller, P. A.; Miller, M. J. J. Am. Chem. Soc. 2012, 134, 9898.

[42] Ji, C.; Miller, M. J. Bioorg. Med. Chem. 2012, 20, 3828.

[43] Miller, M. J.; Zhu, H.; Xu, Y.; Wu, C.; Walz, A. J.; Vergne, A.; Roosenberg, J. M.; Moraski, G.; Minnick, A. A.; Mckee-Dolence, J.; Hu, J.; Fennell, K.; Kurtdolence, E.; Dong, L.; Franzblau, S.; Malouin, F.; Mollmann, U. Biometals 2009, 22, 61.

[44] Cimarusti, C. M.; Sykes, R. B. Med. Res. Rev. 1984, 4, 1.

[45] Bush, K.; Freudenberger, J. S.; Sykes, R.B. Antimicrob. Agents Chem. 1982, 22, 414

[46] Cusnir,R.; Imberti, C.; Hider, R.C.; Blower, P. J.; Ma, M. T. Int. J.
Mol. Sci. 2017, 18, 1161.

[47] Barbachyn, M. R.; Tuominen, T. C. J. Antibiot. 1990, 43, 1199.

[48] Han, S.; Zaniewski, R. P.; Marr, E. S.; Lacey, B. M.; Tomaras, A. P.; Evdokimov, A.; Miller, J. R.; Shanmugasundaram, V. Proc. Natl. Acad. Sci. U. S. A. 2010, 107, 22002.

[49] Flanagan, M. E.; Brickner, S. J.; Lall, M.; Casavant, J.; Deschenes, L.; Finegan, S. M.; George, D. M.; Granskog, K.; Hardink, J. R.; Huband, M. D.; Thuy, H.; Lamb, L.; Marra, A.; Mitton-Fry, M.; Mueller, J. P.; Mullins, L. M.; Noe, M. C.; O'Donnell, J. P.; Pattavina, D.; Penzien, J. B.; Schuff, B. P.; Sun. J.; Whipple, D. A.; Young, J.; Gootz, T. D. ACS Med. Chem. Lett. 2011, 2, 385.

[50] Mcpherson, C. J.; Aschenbrenner, L. M.; Lacey, B. M.; Fahnoe, K. C.; Lemmon, M. M.; Finegan, S. M.; Tadakamalla, B.; O'Donnell, J. P.; Mueller, J. P.; Tomaras, A. P. Antimicrob. Agents Chemother. 2012, 56, 6334.

[51] Tomaras, A. P.; Crandon, J. L.; Mcpherson, C. J.; Nicolau, D. P. Antimicrob. Agents Chemother. 2015, 59, 2439.

[52] Sato, T.; Yamawaki, K. Clin. Infect. Dis 2019, 69, 529.

[53] Page, M. G.; Dantier, C.; Desarbre, E. Antimicrob. Agents Chemother. 2010, 54, 2291.

[54] Sato, T.; Yamawaki, K. Clin. Infect. Dis. 2019, 69, S538.

[55] Yamano, Y.; Nishikawa, T.; Komatsu, Y. Appl. Microbiol. Biotechnol. 1994, 40, 892.

[56] Aoki, T. Yoshizawa, H.; Yamawaki, K.; Yokoo, K.; Sato, J.; Hisakawa, S.; Hasegawa, Y.; Kusano, H.; Sano, M.; Sugimoto, H.; Nishitani, Y.; Sato, T.; Tsuji, M.; Nakamura, R.; Nishikawa, T.; Yamano, Y. Eur. J. Med. Chem. 2018, 155, 847.

[57] Bird, T. G.; Arnould, J. C.; Bertrandie, A.; Jung, F. H. J. Med. Chem. 1992, 35, 2643.

[58] Ghosh, M.; Miller, M. J. Bioorg. Med. Chem. 1995, 3, 1519.

[59] Poras, H.; Kunesch, G.; Barriere, J. C.; Berthaud, N.; Andremont, A. J. Antibiot. 1998, 51, 786 .

[60] Jones, R. N.; Johnson, D. M.; Erwin, M. E. Antimicrob. Agents Chemother. 1996, 40, 720 .

[61] Bassetti, M.; Baguneid, M.; Bouza, E.; Dryden, M.; Nathwani, D.; Wilcox, M. Clin. Microbiol. Infect. 2014, 204, 3.

[62] Mendes, R. E.; Hogan, P. A.; Streit, J. M.; Jones, R. N.; Flamm, R. K. Antimicrob. Agents Chemother. 2015, 59, 2454.

[63] Paulen, A.; Gasser, V.; Hoegy, F.; Perraud, Q.; Pesset, B.; Schalk, I. J.; Mislin, G. L. A. Org. Biomol. Chem. 2015, 13, 11567.

[64] Paulen, A.; Hoegy, F.; Roche, B.; Schalk, I. J.; Mislin, G. L. A. Bioorg. Med. Chem. Lett. 2017, 27, 4867.

[65] Noel, S.; Gasser, V.; Pesset, B.; Hoegy, F.; Rognan, D.; Schalk, I. J.; Mislin, G. L. A. Org. Biomol. Chem. 2011, 9, 8288.

[66] Liu, R.; Miller, P. A.; Vakulenko, S. B.; Stewart, N. K.; Boggess, W. C.; Miller, M. J. J. Med. Chem. 2018, 61, 3845.

[67] Schalk, I. J. J. Med. Chem. 2018, 61, 3842.

[68] Rivault, F.; Liebert, C.; Burger, A.; Hoegy, F.; Abdallah, M. A.; Schalk, I. J.; Mislin, G. L. A. Bioorg. Med. Chem. Lett. 2007, 17, 640 .

[69] Hennard, C.; Truong, Q. C.; Desnottes, J. F.; Paris, J. M.; Moreau, N. J.; Abdallah, M. A. J. Med. Chem. 2001, 44, 2139.

[70] Barrett, J. F.; Bernstein, J. I.; Krause, H. M.; Hilliard, J. J.; Ohemeng, K. A. Anal. Biochem. 1993, 214, 313.

[71] Wencewicz, T. A.; Long, T. E.; Moellmann, U.; Miller, M. J. Bioconjugate Chem. 2013, 24, 473.

[72] Fardeau, S.; Dassonville-Klimpt, A.; Audic, N.; Sasaki, A.; Pillon, M.; Baudrin, E.; Mullie, C.; Sonnet, P. Bioorg. Med. Chem. 2014, 22, 4049.

[73] Milstien, S.; Cohen, L. A. J. Am. Chem. Soc. 1972, 94, 9158.

[74] Ji, C.; Miller, M. J. Bioorg. Med. Chem. 2012, 20, 3828.

[75] Wilhelm, S.; Tommassen, J.; Jaeger, K. E. J. Bacteriol. 1999, 181, 6977.

[76] Zheng, T.; Nolan, E. M. Bioorg. Med. Chem. Lett. 2015, 25, 4987.

[77] Gupta, D.; Gupta, S. V.; Lee, K.; Amidon, G. L. Mol. Pharmaceutics 2009, 6, 1604

[78] Neumann, W.; Sassone-Corsi, M.; Raffatellu, M.; Nolan, E. M. J. Am. Chem. Soc. 2018, 140, 5193. 
[79] Zheng, T.; Bullock, J. L.; Nolan, E. M. J. Am. Chem. Soc. 2012, 134,18388 .

[80] Neumann, W.; Nolan, E. M. J. Biol. Inorg. Chem. 2018, 23, 1025.

[81] Taylor, S. D.; Palmer, M. Bioorg. Med. Chem. 2016, 24, 6253.

[82] Ghosh, M.; Miller, P. A.; Mollmann, U.; Claypool, W. D.; Schroeder, V. A.; Wolter, W. R.; Suckow, M.; Yu, H.; Li, S.; Huang, W.; Zajicek, J.; Miller, M. J. J. Med. Chem. 2017.;60, 4577.

[83] Ghosh, M.; Lin, Y. M.; Miller, P. A.; Mollmann, U.; Boggess, W. C.; Miller, M. J. ACS Infect. Dis. 2018, 4, 1529.

[84] Randall, C. P.; Mariner, K. R.; Chopra, I.; O'Neill, A. J. Antimicrob. Agents Chemother. 2013, 57, 637.

[85] Kraaij, C.; Vos, W. M.; Siezen, R. J.; Kuipers, O. P. Nat. Prod. Rep. 1999, 16, 575

[86] Willey, J. M.; Donk, W.A. Annu. Rev. Microbiol. 2007, 61, 477.

[87] Yoganathan, S.; Sit, C. S.; Vederas, J. C. Org. Biomol. Chem. 2011, 9, 2133.

[88] Wencewicz, T. A.; Mollmann, U.; Long, T. E.; Miller, M. J. Biometals 2009, 22, 633.

[89] Maiden, M. M.; Hunt, A.; Zachos, M. P.; Gibson, J. A.; Hurwitz, M.
E.; Mulks, M. H.; Waters, C. M. Antimicrob. Agents Chemother. 2018, 62, 96.

[90] Heath, R. J.; Rubin, J. R.; Holland, D. R.; Zhang, E.; Snow, M. E.; Rock, C. O. J. Biol. Chem. 1999, 274, 11110.

[91] Bernier, G.; Girijavallabhan, V.; Murray, A.; Niyaz, N.; Ding, P.; Miller, M. J.; Malouin, F. Antimicrob. Agents Chemother. 2005, 49, 241.

[92] Yamamoto, K.; Shiinoki, Y.; Nishio, M.; Matsuda, Y.; Inouye, Y.; Nakamura, S. J. Antibiot. 1990, 43, 1012.

[93] Mollmann, U.; Ghosh, A.; Dolence, E. K.; Dolence, J. A.; Ghosh, M.; Miller, M. J.; Reissbrodt, R. Biometals 1998, 11, 1.

[94] Rivault, F.; Liebert, C.; Burger, A.; Hoegy, F.; Abdallah, M. A.; Schalk, I. J.; Mislin, G. L. Bioorg. Med. Chem. Lett. 2007, 17, 640.

[95] Souto, A.; Montaos, M. A.; Balado, M.; Osorio, C. R.; Rodriguez, J.; Lemos, M. L.; Jimenez, C. Bioorg. Med. Chem. 2013, 21, 295.

[96] Milner, S. J.; Seve, A.; Snelling, A. M.; Thomas, G. H.; Kerr, K. G.; Routledge, A.; Duhme-Klair, A. K. Org. Biomol. Chem. 2013, 11, 3461. 